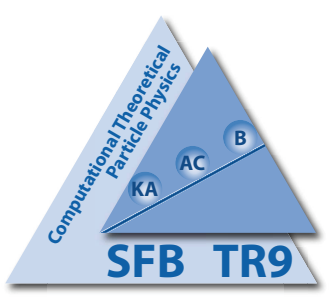

DESY 14-159, DO-TH 14/22, SFB/CPP-14-73, LPN 14-113

\title{
Higher Order Heavy Quark Corrections to Deep-Inelastic Scattering
}

\author{
J. Blümlein ${ }^{a}$, A. De Freitas ${ }^{a, b}$, and C. Schneider ${ }^{b}$ \\ ${ }^{a}$ Deutsches Elektronen-Synchrotron, DESY, Platanenallee 6, D-15738 Zeuthen, Germany \\ ${ }^{b}$ Research Institute for Symbolic Computation (RISC), Johannes Kepler University, Altenbergerstraße 69, A-4040 Linz, Austria
}

\begin{abstract}
The 3-loop heavy flavor corrections to deep-inelastic scattering are essential for consistent next-to-next-to-leading order QCD analyses. We report on the present status of the calculation of these corrections at large virtualities $Q^{2}$. We also describe a series of mathematical, computer-algebraic and combinatorial methods and special function spaces, needed to perform these calculations. Finally, we briefly discuss the status of measuring $\alpha_{s}\left(M_{Z}\right)$, the charm quark mass $m_{c}$, and the parton distribution functions at next-to-next-to-leading order from the world precision data on deepinelastic scattering.
\end{abstract}

Keywords: Deep-inelastic scattering, strong coupling constant, heavy flavor corrections, charm quark mass, mathematical structures

\section{Introduction}

Deep-inelastic scattering (DIS) provides an important method to determine the distribution function of the partons inside nucleons and to measure the strong coupling constant $\alpha_{s}$ from the scaling violations of the nucleon structure functions. Their heavy flavor corrections also allow the determination of the charm quark mass $m_{c}$. Currently the world data on DIS have reached a precision which needs the next-to-next-to-leading order (NNLO) QCD corrections for an adequate description of the data. While the massless 3-loop corrections are known [1-4], the heavy flavor Wilson coefficients are only known to next-to-leading order [5] Currently the NNLO corrections to the heavy flavor Wilson coefficients are being calculated. In this survey we give a detailed account on this project, including technical aspects of the computation, such as systematic analytic summation and integration techniques, and their computer-algebraic implementation. On very

\footnotetext{
${ }^{1}$ For a numerical implementation in Mellin space see [6].
}

general grounds, Feynman integrals may be turned into nested sums [7, 8]. In this context also new mathematical function spaces and algebras are of importance, which have been worked out along with the current project. We also give a brief account on these structures $2^{2}$ Finally, we present recent phenomenological applications determining the twist- 2 parton distribution functions at NNLO in the unpolarized case and at NLO in the polarized case and measuring the strong coupling constant $\alpha_{s}\left(M_{Z}^{2}\right)$ and the charm quark mass $m_{c}$ from the world deep-inelastic scattering data. This survey summarizes main results of the research performed in project B3: 'Structure functions in the continuum' of DFG Sonderforschungsbereich Transregio 9, Computergestützte Theoretische Teilchenphysik. Related studies using lattice gauge methods are given in [11].

The deep-inelastic scattering cross section is of the form

$$
\frac{d^{2} \sigma}{d x d Q^{2}} \sim L^{\mu v} W_{\mu \nu}
$$

\footnotetext{
${ }^{2}$ For recent reviews see [9] 10$]$.
} 
with $x$ the momentum fraction of the parton in the nucleon, $q^{2}=-Q^{2}$ the virtuality of the exchanged gauge boson, and $L^{\mu \nu}$ and $W_{\mu \nu}$ the leptonic and hadronic tensors. While the leptonic tensor is a perturbative quantity, the hadronic tensor is not. In the unpolarized electromagnetic case it is parameterized as [12]

$$
\begin{aligned}
W_{\mu v}= & \frac{1}{4 \pi} \int d^{4} \xi \exp (i q \xi)\langle P, s|\left[J_{\mu}^{\mathrm{em}}(\xi), J_{v}^{\mathrm{em}}(0)|P, s\rangle\right. \\
= & \frac{1}{2 x}\left(g_{\mu \nu}-\frac{q_{\mu} q_{v}}{q^{2}}\right) F_{L}\left(x, Q^{2}\right)+\frac{2 x}{Q^{2}}\left(P_{\mu} P_{v}+\right. \\
& \left.\frac{q_{\mu} P_{v}+q_{v} P_{\mu}}{2 x}-\frac{Q^{2}}{4 x^{2}} g_{\mu v}\right) F_{2}\left(x, Q^{2}\right)
\end{aligned}
$$

The structure functions $F_{2, L}\left(x, Q^{2}\right)$ in 2 are nonperturbative inclusive quantities and contain light and heavy flavor contributions. At sufficiently high values of $Q^{2}$, where the leading twist approximation is valid, the structure functions can be represented by

$$
F_{2, L}\left(x, Q^{2}\right)=\sum_{j} \mathbb{C}_{(2, L), j}\left(x, \frac{Q^{2}}{\mu^{2}}, \frac{m_{i}^{2}}{Q^{2}}\right) \otimes f_{j}\left(x, \mu^{2}\right)
$$

in the $\overline{\mathrm{MS}}$-scheme ${ }^{3}$ Here $\mathbb{C}_{j}$ denote the Wilson coefficients, $\mu$ the factorization scale, $m_{i}$ the heavy quark masses, $f_{j}$ are the twist- 2 parton densities and $\otimes$ is the Mellin convolution

$$
[A \otimes B](x)=\int_{0}^{1} d x_{1} \int_{0}^{1} d x_{2} \delta\left(x-x_{1} x_{2}\right) A\left(x_{1}\right) B\left(x_{2}\right),
$$

which decouples into a product under the Mellin transform

$$
\mathrm{M}[A(x)](N)=\int_{0}^{1} d x x^{N-1} A(x) .
$$

The Wilson coefficients $\mathbb{C}_{j}$ contain both the contributions due to inclusive massless partons, $C_{j}$, as well as the massive quark contributions, $H_{j}$,

$$
\mathbb{C}_{j}=C_{j}+H_{j} .
$$

As has been shown in [14], at large scales $Q^{2} \gg m^{2}$, with $m$ the heavy quark mass, the heavy quark contributions $H_{j}$ factorize into massive operator matrix elements (OMEs) and the massless Wilson coefficients. In the case of the structure function $F_{2}\left(x, Q^{2}\right)$ this factorization applies starting from $Q^{2} / m^{2} \gtrsim 10$, while for the structure function $F_{L}\left(x, Q^{2}\right)$ [15, 16] it applies at much larger scales of $Q^{2} / m^{2}>800$ only [14].

\footnotetext{
${ }^{3}$ For genuine scheme-invariant representations of structure functions see e.g. [13].
}

In the following we review the status of the calculation of the 3-loop QCD corrections of the heavy flavor contributions to the deep-inelastic structure functions at large values of $Q^{2}$ and of the 3-loop matching coefficients in the variable flavor number scheme (VFNS). First we give a survey on the basic relations and discuss the calculation of finite Mellin moments [17]. We turn then to the calculation of these quantities for general values of the Mellin variable $N$, which requires techniques very different from those applied for the individual moments. We first line out different computational methods and discuss then the analytical results having been obtained so far, including numerical predictions. The solution of the corresponding Feynman integrals requests special function spaces which we review together with main relations for these quantities. Finally we give a brief summary on the status of the parton distribution functions (PDFs) both in the unpolarized and polarized case and on the determination of the strong coupling constant $\alpha_{s}\left(M_{Z}\right)$ and the charm quark mass $m_{c}$ using the world DIS and related hard scattering data.

\section{2. $O\left(a_{s}^{3}\right)$ Heavy Flavor Corrections to Deep-Inelastic Scattering}

The leading order QCD heavy flavor corrections to deep inelastic scattering were calculated in Refs. [18] in the later 1970s. They are given by the leading order gauge boson gluon fusion process. In 1992 the nextto-leading order (NLO) corrections were accomplished in Refs. [5] in semi-analytic form and presented for the flavor-tagged process in $x$-space. A numerical implementation of these corrections in Mellin- $N$ has been given in [6]. In the case of the contributions to the inclusive structure functions $F_{2, L}\left(x, Q^{2}\right)$ further virtual corrections contribute, cf. [19].

Fully analytic representations at 2-loop order could be obtained in the region of large virtualities using the factorization [14]. Results were given in the unpolarized [14, 19, 22] and polarized case [23, 24] for neutral current interactions and in the charged current case [25]. The latter results had to be corrected for part of the terms in [26] $]^{4}$ The calculation in this case requests proper current crossing, cf. [29]. As a preparatory step to calculate the 3-loop corrections, the 2-loop OMEs had to be calculated in the dimensional parameter $\varepsilon=D-4$ up to $O(\varepsilon)$ [30], which required more advanced analytic summation technologies.

Let us now turn to the asymptotic heavy flavor Wilson coefficients in the inclusive case and to the OMEs at 3loop order.

\footnotetext{
${ }^{4}$ For the corresponding 1-loop results, see [27, 28].
} 


\subsection{General Formalism}

The Wilson coefficients in the region $Q^{2} \gg m^{2}$ are given by $[17$

$$
\begin{aligned}
L_{2, q}^{\mathrm{NS}}\left(N_{F}\right)= & a_{s}^{2}\left[A_{q q, Q}^{\mathrm{NS},(2)}\left(N_{F}\right)+\hat{C}_{2, q}^{\mathrm{NS},(2)}\left(N_{F}\right)\right]+a_{s}^{3}\left[A_{q q, Q}^{\mathrm{NS},(3)}\left(N_{F}\right)\right. \\
+ & \left.A_{q q, Q}^{\mathrm{NS},(2)}\left(N_{F}\right) C_{2, q}^{\mathrm{NS},(1)}\left(N_{F}\right)+\hat{C}_{2, q}^{\mathrm{NS},(3)}\left(N_{F}\right)\right] \\
\tilde{L}_{2, q}^{\mathrm{PS}}\left(N_{F}\right)= & a_{s}^{3}\left[\tilde{A}_{q q, Q}^{\mathrm{PS},(3)}\left(N_{F}\right)+A_{g q, Q}^{(2)}\left(N_{F}\right) \tilde{C}_{2, g}^{(1)}\left(N_{F}+1\right)\right. \\
+ & \left.\hat{C}_{2, q}^{\mathrm{PS},(3)}\left(N_{F}\right)\right] \\
\tilde{L}_{2, g}^{\mathrm{S}}\left(N_{F}\right)= & a_{s}^{2} A_{g g, Q}^{(1)}\left(N_{F}\right) \tilde{C}_{2, g}^{(1)}\left(N_{F}+1\right)+a_{s}^{3}\left[\tilde{A}_{q g, Q}^{(3)}\left(N_{F}\right)\right. \\
+ & A_{g g, Q}^{(1)}\left(N_{F}\right) \tilde{C}_{2, g}^{(2)}\left(N_{F}+1\right)+A_{g g, Q}^{(2)}\left(N_{F}\right) \tilde{C}_{2, g}^{(1)}\left(N_{F}+1\right) \\
& \left.+A_{Q g}^{(1)}\left(N_{F}\right) \tilde{C}_{2, q}^{\mathrm{PS},(2)}\left(N_{F}+1\right)+\tilde{\tilde{C}}_{2, g}^{(3)}\left(N_{F}\right)\right] \\
= & a_{s}^{2}\left[A_{Q q}^{\mathrm{PS},(2)}\left(N_{F}\right)+\tilde{C}_{2, q}^{\mathrm{PS},(2)}\left(N_{F}+1\right)\right]+a_{s}^{3}\left[A_{Q q}^{\mathrm{PS},(3)}\left(N_{F}\right)\right. \\
+ & \tilde{C}_{2, q}^{\mathrm{PS},(3)}\left(N_{F}+1\right)+A_{g q, Q}^{(2)}\left(N_{F}\right) \tilde{C}_{2, g}^{(1)}\left(N_{F}+1\right) \\
& \left.+A_{Q q}^{\mathrm{PS},(2)}\left(N_{F}\right) C_{2, q}^{\mathrm{NS},(1)}\left(N_{F}+1\right)\right] \\
H_{2, q}^{\mathrm{PS}}\left(N_{F}\right) & a_{s}\left[A_{Q g}^{(1)}\left(N_{F}\right)+\tilde{C}_{2, g}^{(1)}\left(N_{F}+1\right)\right]+a_{s}^{2}\left[A_{Q g}^{(2)}\left(N_{F}\right)\right. \\
+ & A_{Q g}^{(1)}\left(N_{F}\right) C_{2, q}^{\mathrm{NS},(1)}\left(N_{F}+1\right)+A_{g g, Q}^{(1)}\left(N_{F}\right) \tilde{C}_{2, g}^{(1)}\left(N_{F}+1\right) \\
+ & \left.\tilde{C}_{2, g}^{(2)}\left(N_{F}+1\right)\right]+a_{s}^{3}\left[A_{Q g}^{(3)}\left(N_{F}\right)+A_{Q g}^{(2)}\left(N_{F}\right)\right. \\
& \times C_{2, q}^{\mathrm{NS},(1)}\left(N_{F}+1\right)+A_{g g, Q}^{(2)}\left(N_{F}\right) \tilde{C}_{2, g}^{(1)}\left(N_{F}+1\right) \\
+ & A_{Q g}^{(1)}\left(N_{F}\right)\left[C_{2, q}^{\mathrm{NS},(2)}\left(N_{F}+1\right)+\tilde{C}_{2, q}^{\mathrm{PS},(2)}\left(N_{F}+1\right)\right] \\
+ & \left.A_{g g, Q}^{(1)}\left(N_{F}\right) \tilde{C}_{2, g}^{(2)}\left(N_{F}+1\right)+\tilde{C}_{2, g}^{(3)}\left(N_{F}+1\right)\right]
\end{aligned}
$$

Here $a_{s}=\alpha_{s} /(4 \pi)$ denotes the strong coupling constant and we used the convention $\hat{f}\left(N_{F}\right)=f\left(N_{F}+1\right)-$ $\left.f\left(N_{F}\right), \tilde{f}\left(N_{F}\right)=f\left(N_{F}\right) / N_{F}, \hat{\tilde{f}}\left(N_{F}\right)=\tilde{[\tilde{f}}\right]\left(N_{F}\right)$. Since the massless Wilson coefficients are known [1, 4], only the massive OMEs $A_{i j}$ at 3-loop order have to be calculated.

In many applications in collider physics one is interested in describing the process of a heavy quark becoming light at large virtualities. Here one would like to match the universal terms for the parton distributions and their combinations within this transition for one heavy quark turning into an effectively massless state at the time ${ }^{5}$ The relations between the parton distribution functions for $N_{F}$ and $N_{F}+1$ massless quarks, respectively, describing the variable flavor number scheme (VFNS) at a matching scale $\mu^{2}$ read [17, 22]

$$
\begin{aligned}
& f_{k}\left(N_{F}+1\right)+f_{\bar{k}}\left(N_{F}+1\right)= \\
& A_{q q, Q}^{\mathrm{NS}}\left(N_{F}, \frac{\mu^{2}}{m^{2}}\right) \cdot\left[f_{k}\left(N_{F}\right)+f_{\bar{k}}\left(N_{F}\right)\right]+\tilde{A}_{q q, Q}^{\mathrm{PS}}\left(N_{F}, \frac{\mu^{2}}{m^{2}}\right) \cdot \Sigma\left(N_{F}, \mu^{2}\right) \\
& \quad+\tilde{A}_{q g, Q}\left(N_{F}, \frac{\mu^{2}}{m^{2}}\right) \cdot G\left(N_{F}, \mu^{2}\right), \\
& f_{Q}\left(N_{F}\right)+f_{\bar{Q}}\left(N_{F}+1\right)= \\
& A_{Q q}^{\mathrm{PS}}\left(N_{F}, \frac{\mu^{2}}{m^{2}}\right) \cdot \Sigma\left(N_{F}, \mu^{2}\right)+A_{Q g}\left(N_{F}, \frac{\mu^{2}}{m^{2}}, N\right) \cdot G\left(N_{F}, \mu^{2}\right),
\end{aligned}
$$

\footnotetext{
${ }^{5}$ For the corresponding relations in the case of two heavy quarks becoming light, see Ref. 31.
}

$$
\begin{aligned}
& \Sigma\left(N_{F}+1\right)= \\
& {\left[A_{q q, Q}^{\mathrm{NS}}\left(N_{F}, \frac{\mu^{2}}{m^{2}}\right)+N_{F} \tilde{A}_{q q, Q}^{\mathrm{PS}}\left(N_{F}, \frac{\mu^{2}}{m^{2}}\right)+A_{Q q}^{\mathrm{PS}}\left(N_{F}, \frac{\mu^{2}}{m^{2}}\right)\right] \cdot \Sigma\left(N_{F}, \mu^{2}\right)} \\
& +\left[N_{F} \tilde{A}_{q g, Q}\left(N_{F}, \frac{\mu^{2}}{m^{2}}\right)+A_{Q g}\left(N_{F}, \frac{\mu^{2}}{m^{2}}\right)\right] \cdot G\left(N_{F}, \mu^{2}\right),
\end{aligned}
$$

$$
\begin{aligned}
& G\left(N_{F}+1\right)= \\
& A_{g q, Q}\left(N_{F}, \frac{\mu^{2}}{m^{2}}\right) \cdot \Sigma\left(N_{F}, \mu^{2}\right)+A_{g g, Q}\left(N_{F}, \frac{\mu^{2}}{m^{2}}\right) \cdot G\left(N_{F}, \mu^{2}\right), \\
& \Delta\left(N_{F}+1\right)= \\
& f_{k}\left(N_{F}+1, \mu^{2}\right)+f_{\bar{k}}\left(N_{F}+1, \mu^{2}\right)-\frac{1}{N_{F}+1} \Sigma\left(N_{F}+1, \mu^{2}\right),
\end{aligned}
$$

with $k=u, d, s$ denoting the massless quark flavors. We would like to note that usually the appropriate scale $\mu^{2}$ is different from that of the heavy quark mass $m^{2}$ [32].

At the 3-loop level the eight OMEs to be calculated consist of 2964 Feynman diagrams with local operator insertions [33], cf. [17]. They are generated using an extension [17] of QGRAF [34]. The results for the individual diagrams are then written in FORM [35]. The color algebra is carried out using the code Color [36]. In the FORM-code [17] the Dirac-algebra is performed. It has exits both to the calculation of individual moments and for a structure allowing to calculate them for general values of the Mellin variable $N$ in $D$ dimensions. In this way one first obtains the unrenormalized massive OMEs. In the following we will focus on the case of a single heavy quark mass. Recently, also the case of two heavy quark masses has been dealt with in [31]. Let us illustrate the structure of the unrenormalized OME for the flavor pure singlet case [17, 37] as an example :

$$
\begin{aligned}
& \hat{A}_{Q q}^{(3), \mathrm{PS}}= \\
& \left(\frac{\hat{m}^{2}}{\mu^{2}}\right)^{3 \varepsilon / 2}\left[\frac{\hat{\gamma}_{q g}^{(0)} \gamma_{g q}^{(0)}}{6 \varepsilon^{3}}\left(\gamma_{g g}^{(0)}-\gamma_{q q}^{(0)}+6 \beta_{0}+16 \beta_{0, Q}\right)+\frac{1}{\varepsilon^{2}}\left(-\frac{4 \hat{\gamma}_{q q}^{(1), \mathrm{PS}}}{3}\right.\right. \\
& \left.\times\left[\beta_{0}+\beta_{0, Q}\right]-\frac{\gamma_{g q}^{(0)} \hat{\gamma}_{q g}^{(1)}}{3}+\frac{\hat{\gamma}_{q g}^{(0)}}{6}\left[2 \hat{\gamma}_{g q}^{(1)}-\gamma_{g q}^{(1)}\right]+\delta m_{1}^{(-1)} \hat{\gamma}_{q g}^{(0)} \gamma_{g q}^{(0)}\right) \\
& +\frac{1}{\varepsilon}\left(\frac{\hat{\gamma}_{q q}^{(2) \mathrm{PS}}}{3}-N_{F} \frac{\hat{\gamma}_{q q}^{(2), \mathrm{PS}}}{3}+\hat{\gamma}_{q g}^{(0)} a_{g q, Q}^{(2)}-\gamma_{g q}^{(0)} a_{Q g}^{(2)}-4\left(\beta_{0}+\beta_{0, Q}\right) a_{Q q}^{(2), \mathrm{PS}}\right. \\
& \left.-\frac{\hat{\gamma}_{q g}^{(0)} \gamma_{g q}^{(0)} \zeta_{2}}{16}\left[\gamma_{g g}^{(0)}-\gamma_{q q}^{(0)}+6 \beta_{0}\right]+\delta m_{1}^{(0)} \hat{\gamma}_{q g}^{(0)} \gamma_{g q}^{(0)}-\delta m_{1}^{(-1)} \hat{\gamma}_{q q}^{(1), \mathrm{PS}}\right) \\
& \left.+a_{Q q}^{(3), \mathrm{PS}}\right] .
\end{aligned}
$$

Here $\gamma_{i j}^{(k)}$ denote anomalous dimensions, $\beta_{k}$ and $\beta_{k, Q}$ are the expansion coefficients in the massless and massive case, $\delta m_{i}^{(j)}$ are the expansion coefficients of the running mass, and $a_{i j}^{(k)}\left(\bar{a}_{i j}^{(k)}\right)$ are the constant and $O(\varepsilon)$ parts of the lower order unrenormalized OMEs, respectively. From the single pole term $O\left(\varepsilon^{-1}\right)$ the contributions $\propto N_{F}$ of 
the 3-loop anomalous dimensions are obtained as a byproduct of the calculation. In the case of $\gamma_{q q}^{\mathrm{PS}}$ and $\gamma_{q g}$ the complete anomalous dimensions are obtained.

The renormalization of the massive OMEs comprises four steps [17]:

- mass renormalization [38]

- coupling constant renormalization

- renormalization of the local operator

- treatment of the collinear singularities.

The external legs of the OMEs are massless on-shell partons ${ }^{6}$ and the scale of the diagram is set by the heavy quark mass. As mass effects are involved, we treat the coupling renormalization using the background field method first [40], which leads to a MOM scheme [17]. The transition to the $\overline{\mathrm{MS}}$ scheme is performed using the relation

$$
Z_{g}^{\overline{\mathrm{MS}}^{2}}\left(N_{F}+1\right) a_{s}^{\overline{\mathrm{MS}}}\left(\mu^{2}\right)=Z_{g}^{\mathrm{MOM}^{2}}\left(N_{F}+1\right) a_{s}^{\mathrm{MOM}}\left(\mu^{2}\right) .
$$

The $Z$-factors for the ultraviolet and collinear singularities, unlike in the massless case, are here not inverse to each other.

\subsection{Moments}

We first discuss the calculation of finite moments of the different OMEs. They are calculated projecting the results of the FORM-calculation on a massive tadpole, which is then evaluated using the code MATAD [41]. The calculation requests about a factor of 5 more memory and computational time going from $N \rightarrow N+2$. The present resources allow to compute moments up to $N=10, \ldots, 14$ depending on the OME [17]. For transversity [42] corresponding results have been derived in [43]. As a representative of the moments we show the 10th moment of the constant part of the unrenormalized OME $A_{Q g}^{(3)}, a_{Q g}^{(3)}(N=10)$, [17]:

$$
\begin{aligned}
& a_{Q g}^{(3)}(10)= \\
& T_{F} C_{A}^{2}\left(\frac{6830363463566924692253659}{685850575063965696000000}-\frac{563692}{81675} B_{4}+\frac{483988}{9075} \zeta_{4}\right. \\
& \left.-\frac{103652031822049723}{415451499724800} \zeta_{3}-\frac{20114890664357}{581101290000} \zeta_{2}\right) \\
& +T_{F} C_{F} C_{A}\left(\frac{872201479486471797889957487}{2992802509370032128000000}+\frac{1286792}{81675} B_{4}\right.
\end{aligned}
$$

\footnotetext{
${ }^{6}$ External massive on-shell partons have been considered in Ref. [39.
}

$$
\begin{aligned}
& \left.-\frac{643396}{9075} \zeta_{4}-\frac{761897167477437907}{33236119977984000} \zeta_{3}+\frac{15455008277}{660342375} \zeta_{2}\right) \\
& +T_{F} C_{F}^{2}\left(-\frac{247930147349635960148869654541}{148143724213816590336000000}-\frac{11808}{3025} B_{4}\right. \\
& \left.+\frac{53136}{3025} \zeta_{4}+\frac{9636017147214304991}{7122025709568000} \zeta_{3}+\frac{14699237127551}{15689734830000} \zeta_{2}\right) \\
& +T_{F}^{2} C_{A}\left(\frac{23231189758106199645229}{633397356480430080000}+\frac{123553074914173}{5755172290560} \zeta_{3}\right. \\
& \left.+\frac{4206955789}{377338500} \zeta_{2}\right) \\
& +T_{F}^{2} C_{F}\left(-\frac{18319931182630444611912149}{1410892611560158003200000}-\frac{502987059528463}{113048027136000} \zeta_{3}\right. \\
& \left.+\frac{24683221051}{46695639375} \zeta_{2}\right)-\frac{896}{1485} T_{F}^{3} \zeta_{3} \\
& +N_{F} T_{F}^{2} C_{A}\left(\frac{297277185134077151}{15532837481700000}-\frac{1505896}{245025} \zeta_{3}+\frac{189965849}{188669250} \zeta_{2}\right) \\
& +N_{F} T_{F}^{2} C_{F}\left(-\frac{1178560772273339822317}{107642563748181000000}+\frac{62292104}{13476375} \zeta_{3}\right. \\
& \left.+\frac{49652772817}{93391278750} \zeta_{2}\right) .
\end{aligned}
$$

Here $N_{F}$ denotes the number of massless flavors and the color factors are $C_{A}=N_{c}, C_{F}=\left(N_{c}^{2}-1\right) /\left(2 N_{c}\right), T_{F}=$ $1 / 2$ for $S U\left(N_{c}\right)$ and $N_{c}=3$ for $\mathrm{QCD}, \zeta_{k}=$ $\sum_{l=1}^{\infty} 1 / l^{k}, k \geq 2$ denote the values of the Riemann $\zeta$ function at the integers $k$, cf. [44], and the constant $B_{4}$ is given by

$$
B_{4}=-4 \zeta_{2} \ln ^{2}(2)+\frac{2}{3} \ln ^{4}(2)-\frac{13}{2} \zeta_{4}+16 \operatorname{Li}_{4}\left(\frac{1}{2}\right),
$$

and $\operatorname{Li}_{n}(x)=\sum_{k=1}^{\infty} x^{k} / k^{n}$ denotes the polylogarithm [45, 46.

\subsection{Results for general values of $N$}

The renormalized OMEs (15) at 3-loop order have the form

$$
A_{i j}^{(3)}=\sum_{k=0}^{3} \hat{a}_{i j}^{(3,3-k)} \ln ^{k}\left(\frac{m^{2}}{\mu^{2}}\right),
$$

with $\mu^{2}$ the renormalization scale. All logarithmic contributions can be predicted from the 2-loop results and the 3-loop anomalous dimensions, as well as a series of contributions to the constant term. They have been discussed in detail in Ref. [16]. It remains to calculate the constant term of the unrenormalized OMEs. Before we come to the physical results we outline the calculation methods used. 


\subsubsection{Calculation Methods}

In the case of general values of $N$ we have reduced all Feynman diagrams to master integrals by the integration by parts (IBP) relations [47, 48] using an extended version of Reduze2 [49 $]^{7}$ allowing for the treatment of local operators, resumming them into a generating function. We generally refer to this representation in the case of the more involved diagrams, while simpler ones have been calculated directly without any reduction.

The different Feynman integrals or the master integrals are calculated by the following techniques. In the simpler cases they integrate to Beta-functions or (generalized) hypergeometric functions [53, 54], after suitable binomial decompositions and using special variable transformations [55]

$$
\begin{aligned}
& { }_{2} F_{1}(a, b ; c ; x)=\frac{\Gamma(c)}{\Gamma(b) \Gamma(c-b)} \int_{0}^{1} d z z^{b-1}(1-z)^{c-b-1}(1-z x)^{-a} \\
& { }_{p+1} F_{q+1}(c,(a) ; d,(b) ; x)=\frac{\Gamma(d)}{\Gamma(d) \Gamma(d-c)} \int_{0}^{1} d z z^{c-1} \\
& \times(1-z)^{d-c-1} F_{q}((a) ;(b) ; z x) \text {. }
\end{aligned}
$$

More complicated integrals can be mapped to Appell and Kampé de Fériet functions [56]. These functions are represented in terms of multiple sums. Subsequently the expansion in the dimensional parameter $\varepsilon=D-4$ has to be performed. There are special algorithms to perform the $\varepsilon$-expansion under certain conditions [57,-62]. However, for general summand structures the expansion has been built into the package Sigma [63, 64]. The multiply nested infinite and finite sums have to be evaluated. This is done using the packages Sigma based on difference field and ring theory [65-73], EvaluateMultiSum, SumProduction [74] and $\rho$ Sum [75]. The infinite sums need limiting processes and thus require the asymptotic expansion of the respective sums. Furthermore, one would like to map to basis representations. This can be done with the help of the package HarmonicSums [76-79].

In general it turns out that the higher transcendental functions which would be needed in representing the occurring Feynman diagrams at intermediary steps are not found in the literature, but may be represented in terms of a few Mellin-Barnes integrals [80]

$$
\frac{1}{(A+B)^{\alpha}}=\frac{1}{2 \pi i} \int_{c-i \infty}^{c+i \infty} d z A^{z} B^{-\alpha-z} \frac{\Gamma(-z) \Gamma(\alpha+z)}{\Gamma(\alpha)}
$$

\footnotetext{
${ }^{7}$ Reduze2 uses the packages Fermat [50] and Ginac [51]. IBPreductions have also been implemented in the codes [52].
}

using algorithms implemented in [81, 82]. Taking residues one arrives at nested sums, which may again be solved using Sigma.

In some of the cases very large numbers of intermediary nested sums over hypergeometric terms emerge, which leads to very large computation times for Sigma, associated with a large memory request. Since Sigma solves sum by sum, a behaviour of this kind may be related to the fact that several sums (integrals) have to be solved at once. At the side of step-by-step summation, multi-summation algorithms [8, 83] may be applied. We have successfully computed the corresponding integrals using an implementation of the multi-variate AlmkvistZeilberger theorem [79, 84]. It allows for multivariate integrals of the type

$$
\prod_{i=1}^{k} \int_{a_{i}}^{b_{i}} d x_{i}\left(P\left(x_{n}\right)\right)^{N} \prod_{l=1}^{m}\left(p_{l}\left(x_{n}\right)\right)^{r_{l}}, \quad N \in \mathbb{N} \backslash\{0\}, r_{l} \in \mathbb{R}
$$

to derive a difference equation in $N$ with polynomial coefficients in $N$ and $\varepsilon, Q_{k}$,

$$
\sum_{k=0}^{l} Q_{k}(N, \varepsilon) F(N+k)=0 .
$$

Here $P\left(x_{i}\right)$ and $p_{l}\left(x_{i}\right)$ are polynomials and $r_{l}$ will contain the parameter $\varepsilon$. A term $\left(p_{l}\left(x_{i}\right)\right)^{r}$ is called hyperexponential. Sometimes it may be practical to search for inhomogeneous difference equations instead of (24). The corresponding difference equations are solved using Sigma, and sequentially expanding in $\varepsilon$.

A further method consists in deriving systems of linear differential equations for the master integrals [85]. In part they may be obtained by the IBP-relations having been derived before. These systems may be turned into systems of difference equations and solved using the packages Sigma and OreSys [86]. We have also used the method of hyperlogarithms [87], extended to the case of massive Feynman diagrams containing local operator insertions [88, 89], by which a series of diagrams having no poles in $\varepsilon$ can be calculated. Furthermore, guessing methods [90] are helpful to set up difference equations in a series of cases, see also [91].

In complicated cases several of the above methods are combined. It is needless to say that both the direct calculation of different Feynman integrals as well as the calculation of the master integrals may require computer memory in the multi-ten Gbyte region and run-times of several days to weeks.

\subsubsection{3-Loop anomalous dimensions}

The contributions $\propto N_{F}$ of the 3-loop anomalous dimensions can be obtained from the single pole terms of 
the unrenormalized OMEs. Fixed moments have been computed in [17] in the present massive calculation and found to agree with previous results given in [92]. For general values of $N$ we show as one example the result in the pure singlet case

$$
\begin{aligned}
& \gamma_{q q}^{(2), \mathrm{PS}}(N)=\frac{1}{2}\left[1+(-1)^{N}\right]\left\{C _ { F } ^ { 2 } T _ { F } N _ { F } \left\{-\frac{8\left(N^{2}+N+2\right) Q_{1} S_{1}^{2}}{(N-1) N^{3}(N+1)^{3}(N+2)}\right.\right. \\
& +\frac{32 Q_{8} S_{1}}{(N-1) N^{4}(N+1)^{4}(N+2)^{3}}-\frac{8 Q_{12}}{(N-1) N^{5}(N+1)^{5}(N+2)^{3}} \\
& -\frac{8 Q_{6} S_{2}}{(N-1) N^{3}(N+1)^{3}(N+2)^{2}}+32 F\left[\frac{1}{3} S_{1}^{3}-S_{2} S_{1}-\frac{7}{3} S_{3}+2 S_{2,1}\right. \\
& \left.\left.+6 \zeta_{3}\right]\right\}+C_{F} T_{F}^{2} N_{F}^{2}\left\{-\frac{64 Q_{9}}{27(N-1) N^{4}(N+1)^{4}(N+2)^{3}}\right. \\
& \left.+\frac{64 Q_{7} S_{1}}{9(N-1) N^{3}(N+1)^{3}(N+2)^{2}}-F \frac{32}{3}\left[S_{1}^{2}+S_{2}\right]\right\} \\
& +C_{F} C_{A} T_{F} N_{F}\left\{\frac{8\left(N^{2}+N+2\right) Q_{3} S_{1}^{2}}{3(N-1)^{2} N^{3}(N+1)^{3}(N+2)^{2}}\right. \\
& -\frac{16 Q_{11} S_{1}}{9(N-1)^{2} N^{4}(N+1)^{4}(N+2)^{2}}+\frac{16 Q_{13}}{27(N-1)^{2} N^{5}(N+1)^{5}(N+2)^{4}} \\
& +(-1)^{N} \\
& \times\left[\frac{128 Q_{2} S_{1}}{3(N-1) N^{2}(N+1)^{3}(N+2)^{3}}-\frac{128 Q_{10}}{9(N-1) N^{3}(N+1)^{5}(N+2)^{4}}\right] \\
& +\frac{8\left(N^{2}+N+2\right) Q_{4} S_{2}}{3(N-1)^{2} N^{3}(N+1)^{3}(N+2)^{2}}+\frac{32 Q_{5} S_{-2}}{(N-1) N^{3}(N+1)^{3}(N+2)^{2}} \\
& +\frac{16\left(N^{2}+N+2\right)\left(23 N^{2}+23 N+58\right)}{3(N-1) N^{2}(N+1)^{2}(N+2)} S_{3} \\
& +\frac{32\left(N^{2}+N+2\right)\left(7 N^{2}+7 N+10\right) S_{-3}}{(N-1) N^{2}(N+1)^{2}(N+2)} \\
& +\frac{64\left(N^{2}+N+2\right)\left(3 N^{2}+3 N+2\right) S_{-2,1}}{(N-1) N^{2}(N+1)^{2}(N+2)} \\
& \left.\left.+32 F\left[-\frac{1}{3} S_{1}^{3}+S_{2} S_{1}+2 S_{-2} S_{1}-2 S_{2,1}-6 \zeta_{3}\right]\right\}\right\} . \\
& +
\end{aligned}
$$

Here $F$ and $Q_{i}$ denote polynomials in $N$, see [37] and $S_{\vec{a}} \equiv S_{\vec{a}}(N)$ are harmonic sums, cf. 29). Eq. 25] agrees with the result of [3] and has been firstly recalculated in [37]. We also recalculated the contributions $\propto N_{F}$ to $\gamma_{g q}^{(3)}[93], \gamma_{q q}^{(3), N S}$ and for the transversity anomalous dimension $\gamma_{q q}^{(3, \mathrm{NS}, \mathrm{TR})}$ [94]. In the latter case it was the first calculation ab initio, while previously the anomalous dimension had been calculated referring to the principle of maximal transcendentality in [95]. The 2-loop anomalous dimensions [96, 97] are obtained completely. All results agree with the foregoing literature [2, 3, 92, 98,-100].

\subsubsection{The operator matrix element at general values of the Mellin variable $N$}

Complete results for general value of $N$ have been obtained for all the leading $N_{F}$ contributions to the OMEs [101, 102], the bubble topologies [103] and the $O\left(T_{F}^{2}\right)$ contributions to the OME $A_{g g}^{(3)}[104]$. This includes also the asymptotic Wilson coefficients $L_{2, q}^{\mathrm{PS}}$ and $L_{2, g}^{\mathrm{S}}$. While in the former cases all terms can be expressed in terms of nested harmonic sums [105, 106], in the contributions of $O\left(T_{F}^{2}\right)$ [104] also binomially weighted sums [107] contribute. The corresponding term is given by

$$
\left(\begin{array}{c}
2 N \\
N
\end{array}\right) \frac{1}{4^{N}} \sum_{k=1}^{N}\left(\frac{4^{k} S_{1}(k-1)}{k^{2}\left(\begin{array}{c}
2 k \\
k
\end{array}\right)}-7 \zeta_{3}\right) .
$$

The 3-loop OMEs $A_{q q, Q}^{(3), N S}, A_{q q, Q}^{(3), N S, T R}$ and the Wilson coefficient $L_{2, q}^{\mathrm{NS}}$ and the OME $A_{g q, Q}^{(3)}$ have been calculated in Refs. [93, 94] and the pure singlet OME $A_{Q q}^{(3), P S}$ and Wilson coefficient in $H_{2, q}^{\mathrm{PS}}$ in Ref. [37]. In the former cases the nested harmonic sums are sufficient to represent the OMEs and asymptotic Wilson coefficients. In the latter case also generalized harmonic sums [78, 108] contribute. As an example we show the constant part of the unrenormalized massive pure singlet OME at 3-loop order:

$$
\begin{aligned}
& a_{Q q}^{(3), P S}(N)= \\
& C_{F} T_{F}^{2}\left[\frac { 3 2 } { 2 7 ( N - 1 ) ( N + 3 ) ( N + 4 ) ( N + 5 ) } \left(\frac{P_{15}}{N^{3}(N+1)^{2}(N+2)^{2}} S_{2}\right.\right. \\
& -\frac{P_{19}}{N^{3}(N+1)^{3}(N+2)^{2}} S_{1}^{2}+\frac{2 P_{28}}{3 N^{4}(N+1)^{4}(N+2)^{3}} S_{1} \\
& \left.-\frac{2 P_{32}}{9 N^{5}(N+1)^{4}(N+2)^{4}}\right)-\frac{32 P_{3}}{9(N-1) N^{3}(N+1)^{2}(N+2)^{2}} \zeta_{2} \\
& \left.+\left(\frac{32}{27} S_{1}^{3}-\frac{160}{9} S_{1} S_{2}-\frac{512}{27} S_{3}+\frac{128}{3} S_{2,1}+\frac{32}{3} S_{1} \zeta_{2}-\frac{1024}{9} \zeta_{3}\right) F\right] \\
& +C_{F} N_{F} T_{F}^{2}\left[\frac{16 P_{7}}{27(N-1) N^{3}(N+1)^{3}(N+2)^{2}} S_{1}^{2}\right. \\
& +\frac{208 P_{7} S_{2}}{27(N-1) N^{3}(N+1)^{3}(N+2)^{2}}-\frac{32 P_{21} S_{1}}{81(N-1) N^{4}(N+1)^{4}(N+2)^{3}} \\
& +\frac{32 P_{29}}{243(N-1) N^{5}(N+1)^{5}(N+2)^{4}}+\left(-\frac{16}{27} S_{1}^{3}-\frac{208}{9} S_{1} S_{2}-\frac{1760}{27} S_{3}\right. \\
& \left.\left.-\frac{16}{3} S_{1} \zeta_{2}+\frac{224}{9} \zeta_{3}\right) F+\frac{1}{(N-1) N^{3}(N+1)^{3}(N+2)^{2}} \frac{16 P_{7}}{9} \zeta_{2}\right] \\
& +C_{F}^{2} T_{F}\left[\frac{32 P_{9} S_{2,1}}{3(N-1) N^{3}(N+1)^{3}(N+2)^{2}}\right. \\
& +\frac{16 P_{14} S_{3}}{9(N-1) N^{3}(N+1)^{3}(N+2)^{2}}-\frac{4 P_{17} S_{1}^{2}}{3(N-1) N^{4}(N+1)^{4}(N+2)^{3}} \\
& +\frac{4 P_{23} S_{2}}{3(N-1) N^{4}(N+1)^{4}(N+2)^{3}}+\frac{4 P_{31}}{3(N-1) N^{6}(N+1)^{6}(N+2)^{4}} \\
& +\left(\left(\frac{2 P_{5}}{N^{2}(N+1)^{2}}-\frac{4 P_{1}}{N(N+1)} S_{1}\right) \zeta_{2}-\frac{4 P_{1}}{9 N(N+1)} S_{1}^{3}\right) G \\
& +\left(\left(\frac{80}{9} S_{3}-64 S_{2,1}\right) S_{1}-\frac{2}{9} S_{1}^{4}-\frac{20}{3} S_{1}^{2} S_{2}+\frac{46}{3} S_{2}^{2}+\frac{124}{3} S_{4}\right. \\
& +\frac{416}{3} S_{2,1,1}+64\left(\left(S_{3}(2)-S_{1,2}(2,1)+S_{2,1}(2,1)\right.\right. \\
& \left.-S_{1,1,1,1}(2,1,1)\right) S_{1}\left(\frac{1}{2}\right)-S_{1,3}\left(2, \frac{1}{2}\right)+S_{2,2}\left(2, \frac{1}{2}\right)-S_{3,1}\left(2, \frac{1}{2}\right) \\
& +S_{1,1,2}\left(2,1, \frac{1}{2}\right)-S_{1,2,1}\left(2, \frac{1}{2}, 1\right) \\
& +1)
\end{aligned}
$$




$$
\begin{array}{ll}
+S_{1,2,1}\left(2,1, \frac{1}{2}\right)-S_{2,1,1}\left(2, \frac{1}{2}, 1\right)-S_{2,1,1}\left(2,1, \frac{1}{2}\right) & -\frac{8 P_{18} S_{-2}}{3(N-1) N^{4}(N+1)^{4}(N+2)^{3}}-\frac{4 P_{25} \zeta_{2}}{9(N-1)^{2} N^{4}(N+1)^{4}(N+2)^{3}} \\
\left.+S_{1,1,1,1}\left(2, \frac{1}{2}, 1,1\right)+S_{1,1,1,1}\left(2,1, \frac{1}{2}, 1\right)+S_{1,1,1,1}\left(2,1,1, \frac{1}{2}\right)\right) & \left.-\frac{8 P_{20} \zeta_{3}}{9(N-1)^{2} N^{3}(N+1)^{3}(N+2)^{2}}\right] .
\end{array}
$$$$
\left.+\left(12 S_{2}-4 S_{1}^{2}\right) \zeta_{2}+\left(\frac{112}{3} S_{1}-448 S_{1}\left(\frac{1}{2}\right)\right) \zeta_{3}+144 \zeta_{4}-32 B_{4}\right) F
$$$$
+\frac{32 P_{2} 2^{-N}}{(N-1) N^{3}(N+1)^{2}}\left(-S_{3}(2)+S_{1,2}(2,1)-S_{2,1}(2,1)\right.
$$$$
\left.+S_{1,1,1}(2,1,1)+7 \zeta_{3}\right)+\left(-\frac{4 P_{8}}{3(N-1) N^{3}(N+1)^{3}(N+2)^{2}} S_{2}\right.
$$$$
\left.\left.+\frac{8 P_{27}}{3(N-1) N^{5}(N+1)^{5}(N+2)^{4}}\right) S_{1}+\frac{4 P_{16} \zeta_{3}}{3(N-1) N^{3}(N+1)^{3}(N+2)^{2}}\right]
$$$$
+C_{A} C_{F} T_{F}
$$$$
\times\left[-\frac{8 P_{10} S_{2,1}}{3(N-1) N^{3}(N+1)^{3}(N+2)^{2}}+\frac{8 P_{12} S_{-3}}{3(N-1) N^{3}(N+1)^{3}(N+2)^{2}}\right.
$$$$
+\frac{16 P_{13} S_{-2,1}}{3(N-1) N^{3}(N+1)^{3}(N+2)^{2}}+\frac{8 P_{22} S_{3}}{27(N-1)^{2} N^{3}(N+1)^{3}(N+2)^{2}}
$$$$
-\frac{4\left(P_{24} S_{1}^{2}+P_{26} S_{2}\right)}{27(N-1)^{2} N^{4}(N+1)^{4}(N+2)^{3}}
$$$$
-\frac{8 P_{33}}{243(N-1)^{2} N^{6}(N+1)^{6}(N+2)^{5}}
$$$$
+\left(\frac{4 P_{4} S_{1}^{3}}{27(N-1) N(N+1)(N+2)}+\left(\frac{8}{9}\left(137 N^{2}+137 N+334\right) S_{3}\right.\right.
$$$$
\left.-\frac{16}{3}\left(35 N^{2}+35 N+18\right) S_{-2,1}\right) S_{1}+\frac{8}{3}\left(69 N^{2}+69 N+94\right) S_{-3} S_{1}
$$$$
+\frac{64}{3}\left(7 N^{2}+7 N+13\right) S_{-2} S_{2}+\frac{2}{3}\left(29 N^{2}+29 N+74\right) S_{2}^{2}
$$$$
+\frac{4}{3}\left(143 N^{2}+143 N+310\right) S_{4}-\frac{16}{3}\left(3 N^{2}+3 N-2\right) S_{-2}^{2}
$$$$
+\frac{16}{3}\left(31 N^{2}+31 N+50\right) S_{-4}-8\left(7 N^{2}+7 N+26\right) S_{3,1}
$$$$
-64\left(3 N^{2}+3 N+2\right) S_{-2,2}-\frac{32}{3}\left(23 N^{2}+23 N+22\right) S_{-3,1}
$$$$
+\frac{64}{3}\left(13 N^{2}+13 N+2\right) S_{-2,1,1}+\frac{4 P_{4}}{3(N-1) N(N+1)(N+2)} S_{1} \zeta_{2}
$$$$
\left.-\frac{8}{3}\left(11 N^{2}+11 N+10\right) S_{1} \zeta_{3}\right) G+\left(\frac{112}{3} S_{-2} S_{1}^{2}+\frac{2}{9} S_{1}^{4}+\frac{68}{3} S_{1}^{2} S_{2}\right.
$$$$
-\frac{80}{3} S_{2,1,1}+32\left(\left(-S_{3}(2)+S_{1,2}(2,1)-S_{2,1}(2,1)+S_{1,1,1}(2,1,1)\right)\right.
$$$$
\times S_{1}\left(\frac{1}{2}\right)+S_{1,3}\left(2, \frac{1}{2}\right)-S_{2,2}\left(2, \frac{1}{2}\right)+S_{3,1}\left(2, \frac{1}{2}\right)
$$$$
-S_{1,1,2}\left(2, \frac{1}{2}, 1\right)+S_{1,1,2}\left(2,1, \frac{1}{2}\right)+S_{1,2,1}\left(2, \frac{1}{2}, 1\right)
$$$$
-S_{1,2,1}\left(2,1, \frac{1}{2}\right)+S_{2,1,1}\left(2, \frac{1}{2}, 1\right)+S_{2,1,1}\left(2,1, \frac{1}{2}\right)
$$$$
\left.-S_{1,1,1,1}\left(2, \frac{1}{2}, 1,1\right)-S_{1,1,1,1}\left(2,1, \frac{1}{2}, 1\right)-S_{1,1,1,1}\left(2,1,1, \frac{1}{2}\right)\right)
$$$$
\left.+\left(4 S_{1}^{2}+12 S_{2}+24 S_{-2}\right) \zeta_{2}+224 S_{1}\left(\frac{1}{2}\right) \zeta_{3}-144 \zeta_{4}+16 B_{4}\right) F
$$$$
+\frac{16 P_{2} 2^{-N}}{(N-1) N^{3}(N+1)^{2}}\left(S_{3}(2)-S_{1,2}(2,1)+S_{2,1}(2,1)\right.
$$$$
\left.-S_{1,1,1}(2,1,1)-7 \zeta_{3}\right)+S_{1}\left(\frac{4 P_{11} S_{2}}{9(N-1)^{2} N^{3}(N+1)^{3}(N+2)^{2}}\right.
$$$$
\left.+\frac{4 P_{30}}{81(N-1)^{2} N^{5}(N+1)^{5}(N+2)^{4}}\right)+\frac{32 P_{6} S_{1} S_{-2}}{3(N-1) N^{3}(N+1)^{3}(N+2)^{2}}
$$

Here $G$ and $P_{i}$ denote polynomials in $N$ [37] and $S_{\vec{a}}(\vec{b})$ are generalized harmonic sums, cf. (31). In $x$-space the above contributions due to generalized harmonic sums transform into generalized harmonic polylogarithms [78]. Their specific combination can be reduced to usual harmonic polylogarithms $H_{\vec{a}}$ [109] of argument $1-2 x$. In the case of the non-singlet contributions [94] the general $N$ result has also been calculated in the polarized case, which is possible solving the $\gamma_{5}$-problem via the Ward-Takahashi identity [110].

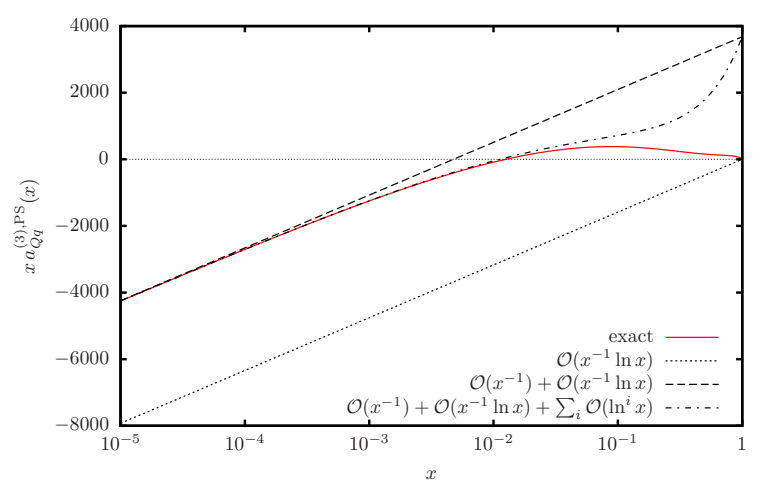

Figure 1: $x a_{Q q}^{(3), \mathrm{PS}}(x)$ in the low $x$ region (solid red line) and leading terms approximating this quantity; dotted line: 'leading' small $x$ approximation $O(\ln (x) / x)$, dashed line: adding the $O(1 / x)$-term, dashdotted line: adding all other logarithmic contributions; from [37.

Let us consider the constant part to the unrenormalized OME $A_{Q q}^{\mathrm{PS}}$ in $x$-space shown in Figure 1, The leading small- $x$ contribution can be determined under some assumptions [111] (for a discussion see [37]), based on an earlier result in Ref. [112]. Our explicit calculation confirms this leading order prediction. However, it describes the function $a_{Q q}^{(3), P S}$ nowhere. The next term in the small- $x$ expansion [37] yields a good description at $x \simeq 10^{-4}$ and many more sub-leading terms have to be added to cover the small- $x$ regime, which is a quite common observation in the case of many quantities [113, 114].

The calculation of the OMEs $A_{g g, Q}^{(3)}$ and $A_{Q g}^{(3)}$, as well as of the Wilson coefficient $H_{2, g}^{\mathrm{S}}$ is currently underway.

We now turn to phenomenological applications and consider the contributions of the completed massive Wilson coefficients in the asymptotic region to the structure function $F_{2}\left(x, Q^{2}\right)$. In Figure 2 the $O\left(a_{s}^{2}\right)$ contribution by $L_{g, 2}^{\mathrm{S}}$ is shown. 


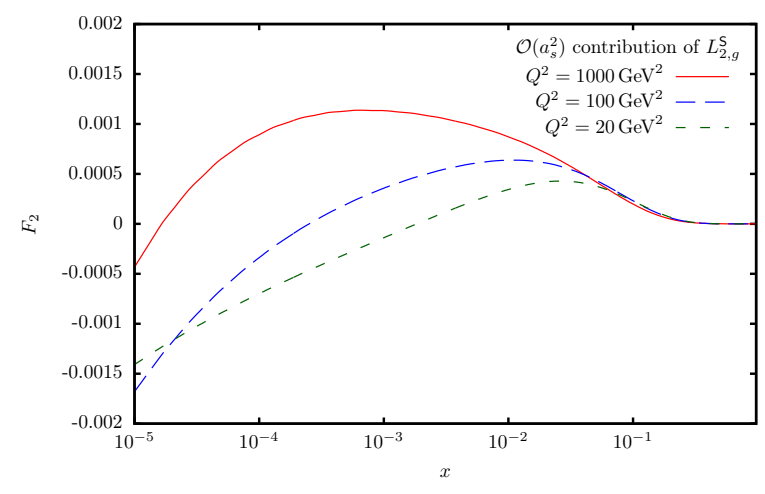

Figure 2: The $O\left(a_{s}^{2}\right)$ contribution by $L_{g, 2}^{\mathrm{S}}$ to the structure function $F_{2}\left(x, Q^{2}\right)$ using the parton distribution functions [115] and $m_{c}=$ $1.59 \mathrm{GeV}$; from [16].

Comparing to the contribution of the Wilson coefficient in 3-loop order, depicted in Figure 3 it turns out that the 3-loop contribution is larger than the 2-loop contribution, which is due to a newly arising small $x$ term.

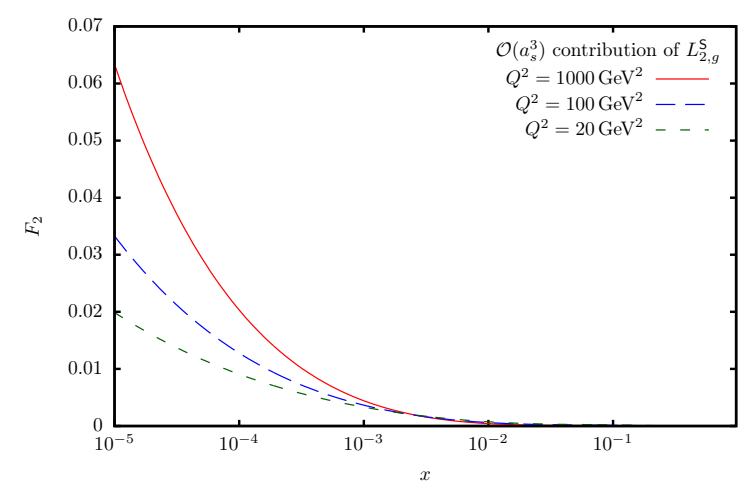

Figure 3: The $O\left(a_{s}^{3}\right)$ contribution by $L_{g, 2}^{\mathrm{S}}$ to the structure function $F_{2}\left(x, Q^{2}\right)$ using the parton distribution functions [115] and $m_{c}=$ $1.59 \mathrm{GeV}$; from [16].

In the physical region of HERA, the widest having been explored so far, $x \geq Q^{2} / S, S \simeq 10^{5} \mathrm{GeV}^{2}$, the corrections are $O(0.01)$ and below. One should keep in mind that the experimental error of the $F_{2}$-data at HERA is of the same size [116]. Furthermore, measurements at the planned facilities EIC [117, 118] and LHeC [119], operating at high luminosities, will have even smaller experimental errors. The pure singlet contributions due to $L_{q, 2}^{\mathrm{PS}}$ shown in Figure 4 are smaller than those of $L_{g, 2}^{\mathrm{S}}$.

The next terms are those due to $L_{q, 2}^{\mathrm{NS}}$ depicted in Figure 5. Both the inclusive heavy flavor 2- and 3-loop corrections are negative. The 3-loop corrections enlarge the effect towards small values of $x$. In the kinematic region at HERA the effects are below $\sim 0.005$.

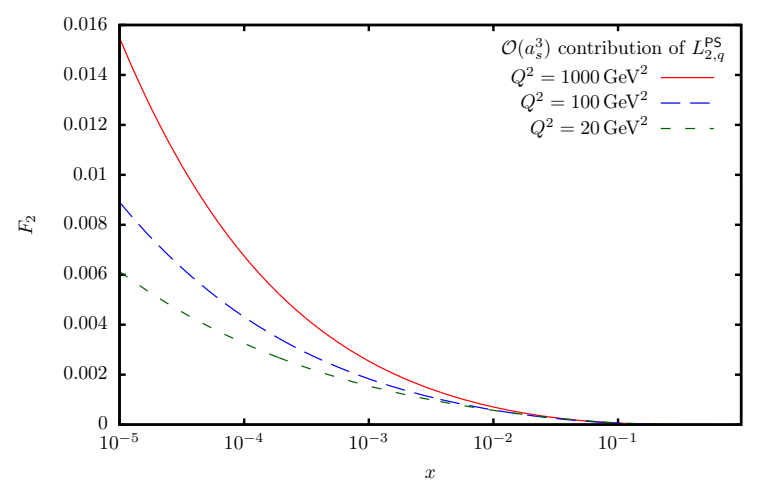

Figure 4: The $O\left(a_{s}^{3}\right)$ contribution by $L_{q, 2}^{\mathrm{PS}}$ to the structure function $F_{2}\left(x, Q^{2}\right)$ using the parton distribution functions [115] and $m_{c}=$ $1.59 \mathrm{GeV}$; from [16].

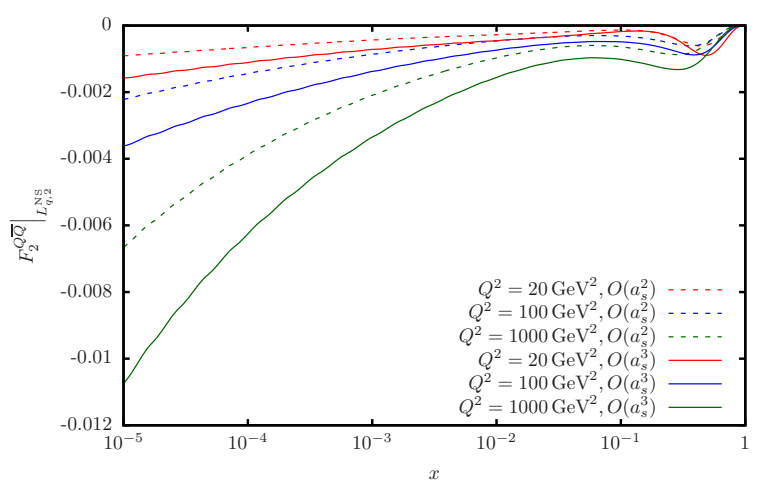

Figure 5: The flavor non-singlet contribution of the Wilson coefficient $L_{q, 2}^{\mathrm{NS}}$ to the structure function $F_{2}\left(x, Q^{2}\right)$ at the 2- and up to the 3-loop order using the NNLO parton distribution functions of Ref. [115] in the on-shell scheme for $m_{c}=1.59 \mathrm{GeV}$. Here we do not display the $O\left(a_{s}^{0}\right)$ terms; from 94 .

In the flavor non-singlet case all OMEs contributing to the matching relation in the VFNS up to 3-loop order (12) have been calculated. It is illustrated in Figure 6 for 2 - and 3-loop order in dependence of the matching scale $Q^{2}$. While at 2-loop order the corrections are very small in the low $x$ region, it grows to large $x$ to up to $\mathrm{O}(0.005)$. The 3-loop corrections are larger due to the gluonic and singlet corrections and vary between negative and positive values of $\mathrm{O}(0.005)$ with more pronounced profiles, also in the small $x$ region.

Finally, the heavy flavor pure singlet contributions to $F_{2}\left(x, Q^{2}\right)$ are illustrated in Figure 7. The corrections are negative both at 2- and 3-loop order and grow with $Q^{2}$ towards small values of $x$. In the kinematic range of 
HERA the corrections amount to $O(-0.03)$.

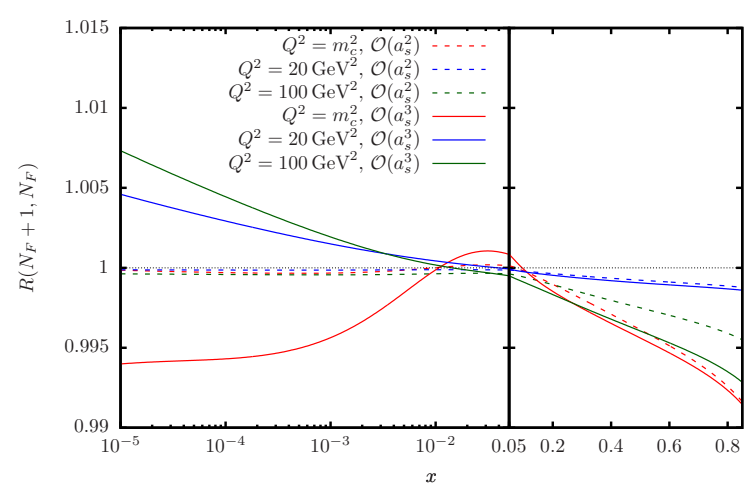

Figure 6: The ratio of the distribution $x(u+\bar{u})$ for four and three quark flavors at 2- and 3-loop order in the variable flavor number scheme matched at different scales of $Q^{2}$ as a function of $x$ using the parton distribution functions of Ref. [115] and the on-mass-shell definition of the charm quark mass $m_{c}=$ $1.59 \mathrm{GeV}$; from [94].

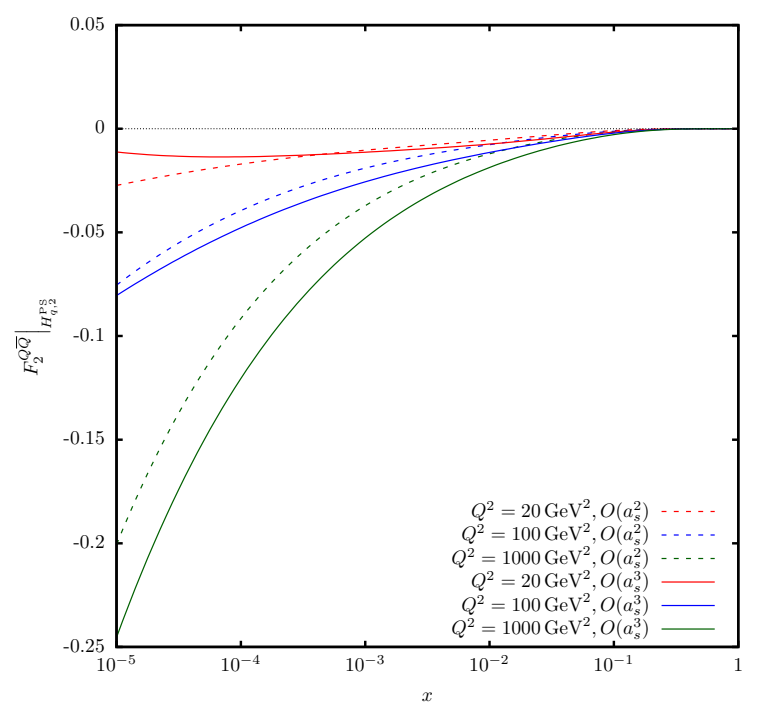

Figure 7: The charm contribution by the Wilson coefficient $H_{Q, 2}^{\mathrm{PS}}$ to the structure function $F_{2}\left(x, Q^{2}\right)$ as a function of $x$ and $Q^{2}$ choosing $Q^{2}=\mu^{2}, m_{c}=1.59 \mathrm{GeV}$ (on-shell scheme) using the parton distribution functions [37].

\subsubsection{The case of two different heavy quark masses}

Beginning with 3-loop order Feynman integrals with two massive internal fermion lines contribute, which give rise to non-factorizable heavy flavor effects. In particular these diagrams cannot be attributed to a single heavy quark species. Due to the fact that $m_{c}^{2} / m_{b}^{2} \sim 1 / 10$, charm will not have decoupled to a massless fermion at the scale of $m_{b}$. The VFNS generalizes in this case and one has to decouple charm and bottom at large scales together rather than individually [31]. Also a series of moments for all OMEs in the 2-mass case has been calculated. The computation proceeds along similar lines as in the single mass case. The corresponding tadpoles are calculated using the code qexp [120]. As an example we show the 6th moment of the constant part of the unrenormalized OME $A_{Q g}^{(3)}$ with $x=m_{1}^{2} / m_{2}^{2}$, expanding in the mass ratio $x$,

$a_{Q g}^{(3)}(N=6)=$

$\frac{1}{2}\left\{T_{F}^{2} C_{A}\left\{\frac{69882273800453}{367569090000}-\frac{395296}{19845} \zeta_{3}+\frac{1316809}{39690} \zeta_{2}\right.\right.$

$+\frac{832369820129}{14586075000} x+\frac{1511074426112}{624023544375} x^{2}-\frac{84840004938801319}{690973782403905000} x^{3}$

$+\ln \left(\frac{m_{2}^{2}}{\mu^{2}}\right)\left[\frac{11771644229}{194481000}+\frac{78496}{2205} \zeta_{2}-\frac{1406143531}{69457500} x\right.$

$\left.-\frac{105157957}{180093375} x^{2}+\frac{2287164970759}{7669816654500} x^{3}\right]+\ln ^{2}\left(\frac{m_{2}^{2}}{\mu^{2}}\right)\left[\frac{2668087}{79380}\right.$

$\left.+\frac{112669}{661500} x-\frac{49373}{51975} x^{2}-\frac{31340489}{34054020} x^{3}\right]+\ln ^{3}\left(\frac{m_{2}^{2}}{\mu^{2}}\right) \frac{324148}{19845}$

$+\ln ^{2}\left(\frac{m_{2}^{2}}{\mu^{2}}\right) \ln \left(\frac{m_{1}^{2}}{\mu^{2}}\right) \frac{156992}{6615}+\ln \left(\frac{m_{2}^{2}}{\mu^{2}}\right) \ln \left(\frac{m_{1}^{2}}{\mu^{2}}\right)\left[\frac{128234}{3969}-\frac{112669}{330750} x\right.$

$\left.+\frac{98746}{51975} x^{2}+\frac{31340489}{17027010} x^{3}\right]+\ln \left(\frac{m_{2}^{2}}{\mu^{2}}\right) \ln ^{2}\left(\frac{m_{1}^{2}}{\mu^{2}}\right) \frac{68332}{6615}+\ln \left(\frac{m_{1}^{2}}{\mu^{2}}\right)[$

$\frac{83755534727}{583443000}+\frac{78496}{2205} \zeta_{2}+\frac{1406143531}{69457500} x+\frac{105157957}{180093375} x^{2}$

$\left.-\frac{2287164970759}{7669816654500} x^{3}\right]+\ln ^{2}\left(\frac{m_{1}^{2}}{\mu^{2}}\right)\left[\frac{2668087}{79380}+\frac{112669}{661500} x-\frac{49373}{51975} x^{2}\right.$

$\left.\left.-\frac{31340489}{34054020} x^{3}\right]+\ln ^{3}\left(\frac{m_{1}^{2}}{\mu^{2}}\right) \frac{412808}{19845}\right\}+T_{F}^{2} C_{F}\left\{-\frac{3161811182177}{71471767500}\right.$

$+\frac{447392}{19845} \zeta_{3}+\frac{9568018}{4862025} \zeta_{2}-\frac{64855635472}{2552563125} x+\frac{1048702178522}{97070329125} x^{2}$

$+\frac{1980566069882672}{2467763508585375} x^{3}+\ln \left(\frac{m_{2}^{2}}{\mu^{2}}\right)\left[\frac{1786067629}{204205050}-\frac{111848}{15435} \zeta_{2}\right.$

$\left.-\frac{128543024}{24310125} x-\frac{22957168}{3361743} x^{2}-\frac{2511536080}{2191376187} x^{3}\right]$

$+\ln ^{2}\left(\frac{m_{2}^{2}}{\mu^{2}}\right)\left[\frac{3232799}{4862025}+\frac{752432}{231525} x+\frac{177944}{40425} x^{2}+\frac{127858928}{42567525} x^{3}\right]$

$-\ln ^{3}\left(\frac{m_{2}^{2}}{\mu^{2}}\right) \frac{111848}{19845}-\ln ^{2}\left(\frac{m_{2}^{2}}{\mu^{2}}\right) \ln \left(\frac{m_{1}^{2}}{\mu^{2}}\right) \frac{223696}{46305}+\ln \left(\frac{m_{2}^{2}}{\mu^{2}}\right) \ln \left(\frac{m_{1}^{2}}{\mu^{2}}\right)$

$\times\left[\frac{22238456}{4862025}-\frac{1504864}{231525} x-\frac{355888}{40425} x^{2}-\frac{255717856}{42567525} x^{3}\right]+\ln \left(\frac{m_{2}^{2}}{\mu^{2}}\right)$

$\ln ^{2}\left(\frac{m_{1}^{2}}{\mu^{2}}\right) \frac{223696}{46305}+\ln \left(\frac{m_{1}^{2}}{\mu^{2}}\right)\left[-\frac{24797875607}{1021025250}-\frac{111848}{15435} \zeta_{2}\right.$

$\left.+\frac{128543024}{24310125} x+\frac{22957168}{3361743} x^{2}+\frac{2511536080}{2191376187} x^{3}\right]$

$+\ln ^{2}\left(\frac{m_{1}^{2}}{\mu^{2}}\right)\left[\frac{3232799}{4862025}+\frac{752432}{231525} x+\frac{177944}{40425} x^{2}+\frac{127858928}{42567525} x^{3}\right\}$

$\left.-\ln ^{3}\left(\frac{m_{1}^{2}}{\mu^{2}}\right) \frac{1230328}{138915}\right\}+O\left(x^{4} \ln ^{3}(x)\right)$. 
The scalar integrals contributing to all topologies of $A_{g g}^{(3)}$ in the 2-mass case at general values of $N$ have also been calculated, which can be expressed in terms of generalized harmonic sums, where no expansion in the mass ratio is performed. Indeed the expansion is possible fixing the value of $N$. Since the expansion leads to problems at general values of $N$ one is forced to seek the complete solution.

\section{Mathematical Aspects of Higher Loop Calcula- tions}

Feynman integrals have representations in special number and function spaces, which obey a sequential order growing with the complexity of the integrals defined by their loop order, the number of legs and scales being involved. This has been observed in various calculations up to two-loop order and inclusive $2 \rightarrow 2$ processes, mainly in QCD, until the mid 1990s, cf. e.g. [121, 122].

For zero scale quantities, like the expansion coefficients of the $\beta$-function or individual moments of operator matrix elements, one obtains a representation over special numbers. Single scale quantities, like splitting functions or Wilson coefficients in deep-inelastic scattering or for the Drell-Yan process lead to 1-dimensional function representations [123, 124].

Not much is known on basis representations in more complex cases. The beginning of a systematic search for basis representations falls in about this time [105. 106, 125, 126] after it has been recognized that using the available function representations [45, 46, 127,-130] contained too complicated arguments for further integration or were even not complete. In the case of zero scale quantities at lower loop order in the massless case, multiple zeta values [44] are sufficient to represent the results. In massive calculations at higher loop order also cyclotomic zeta values [77, 131], generalized infinite sums [78] and constants associated to nested (inverse) binomial sums [107, 132] occur. Zero scale quantities can be obtained as a fixed moment or in the limit $N \rightarrow \infty$ of convergent single scale quantities in Mellin space or as special values of the associated iterated integrals at $x=1$ or other (algebraic) arguments.

The harmonic sums are defined by [105, 106]

$$
S_{b, \vec{a}}(N)=\sum_{k=1}^{N} \frac{(\operatorname{sign}(b))^{k}}{k^{|b|}} S_{\vec{a}}(k), \quad S_{\emptyset}=1, \quad b, a_{i} \in \mathbb{Z} \backslash\{0\} .
$$

They have several generalizations. First one may define the real representations of cyclotomic sums by [77]

$$
S_{b_{1}, b_{2}, b_{3} ; \overrightarrow{\mathbf{a}},}(N)=\sum_{k=1}^{N} \frac{\left(\operatorname{sign}\left(b_{3}\right)\right)^{k}}{\left(b_{1} k+b_{2}\right)^{\left|b_{3}\right|}} S_{\overrightarrow{\mathbf{a}}}(k), \quad S_{\emptyset}=1,
$$

$$
\mathbf{a}=\left(c_{1}, c_{2}, c_{3}\right) ; b_{1}, c_{1} \in \mathbb{N} \backslash\{0\}, b_{2}, c_{2} \in \mathbb{N}, b_{3}, c_{3} \in \mathbb{Z} \backslash\{0\} .
$$

The generalized (cyclotomic) sums are given by $\left[788^{8}\right.$

$$
\begin{aligned}
& S_{b_{1}, b_{2}, b_{3} ; \overrightarrow{\mathbf{a}},}(y, \vec{x} ; N)=\sum_{k=1}^{N} \frac{y^{k}}{\left(b_{1} k+b_{2}\right)^{\left|b_{3}\right|}} S_{\overrightarrow{\mathbf{a}}}(\vec{x} ; k), S_{\emptyset}=1, \\
& x_{i}, y \in \mathbb{C} \backslash\{0\}, \mathbf{a}=\left(c_{1}, c_{2}, c_{3}\right) ; b_{1}, c_{1} \in \mathbb{N} \backslash\{0\}, b_{2}, c_{2} \in \mathbb{N}, \\
& b_{3}, c_{3} \in \mathbb{Z} \backslash\{0\} .
\end{aligned}
$$

They generalize the multiple polylogarithms studied in [126, 133]. Even further generalizations contain the binomial coefficient $\left(\begin{array}{c}2 k \\ k\end{array}\right)$ as a weight factor either in the numerator or denominator of the respective partial sum(s) [107. An example is given by

$$
\sum_{i=1}^{N} \frac{1}{(i+1)\left(\begin{array}{c}
2 i \\
i
\end{array}\right)} \sum_{j=1}^{i}\left(\begin{array}{c}
2 j \\
j
\end{array}\right) \frac{1}{j} S_{-2}(j) .
$$

The sums obey quasi-shuffle relations, i.e. their products are spanned by linear combinations of sums of the same class and polynomials of sums of lower depth [134]. These relations depend only on the sums' index pattern, not on their value and are also called algebraic relations [135]. The linear combination is a sum over all combinations of combined indices, which preserve the order of indices in the factors. For harmonic sums one obtains e.g.

$$
\begin{aligned}
S_{c} S_{a, b} & =S_{c, a, b}+S_{a, c, b}+S_{a, b, c}-S_{c \wedge a, c}-S_{a, x \wedge b},(33) \\
a \wedge b & =\operatorname{sign}(a) \operatorname{sign}(b)(|a|+|b|)
\end{aligned}
$$

Similar quasi shuffle relations are obtained by the cyclotomic [77], generalized [78, 108] and (inverse) binomial harmonic sums [107]. All these nested sums also obey structural relations, which depend on their specific structure beyond their index set. They are implied by multiple integer argument and differentiation. The latter operation leads to equivalence classes of sums. The structural relations have been worked out in Refs. [7, 136] in the case of the harmonic sums and in [77, 78] the cyclotomic and generalized (cyclotomic) sums.

One may associate iterated integrals to all these sums which are obtained by the Mellin transform

$$
\operatorname{M}[f(x)](N)=\int_{0}^{1} d x x^{N-1} f(x) .
$$

\footnotetext{
${ }^{8}$ The generalized harmonic sums without cyclotomy, i.e. for $b_{2}, c_{2}=0$ were given in [108].
} 
In this way an alphabet of letters will be created, defining the respective Mellin transform linearly. In the case of the harmonic sums the alphabet is formed by

$$
\mathfrak{A}=\left\{\frac{1}{x}, \frac{1}{1-x}, \frac{1}{1+x}\right\}
$$

yielding the harmonic polylogarithms [109]

$$
H_{b, \vec{a}}(x)=\int_{0}^{x} d x f_{b}(x) H_{\vec{a}}(x), \quad H_{\emptyset}(x)=1, f_{c}(x) \in \mathfrak{A} .
$$

The alphabets corresponding to the cyclotomic polylogarithms extend $\mathfrak{A}$ adding the inverse of the higher cyclotomic polynomials, i.e.

$$
\left\{\frac{1}{1+x^{2}}, \frac{1}{1-x+x^{2}}, \frac{1}{1+x+x^{2}+x^{3}+x^{4}}, \ldots\right\} \text {. }
$$

In addition, a series of numerator powers $x^{i}$ occur [77]. In the case of the generalized sums the new letters are [78]

$$
\left\{\frac{1}{x-a_{i}}\right\}, a_{i} \in \mathbb{R} \backslash\{0\} .
$$

One may even consider a combination of cyclotomic and generalized harmonic polylogarithms, as implied by the corresponding sums [78]. The nested (inverse) binomial sums imply square-root valued letters like

$$
\left\{\frac{1}{\sqrt{x} \sqrt{1 \pm x}}, \frac{1}{x \sqrt{1 \pm x}}, \frac{1}{\sqrt{2-x} \sqrt{1-x}}, \ldots\right\} .
$$

The special numbers associated to these classes of sums and iterated integrals are obtained in the limit $N \rightarrow \infty$ and for $x=1$, whenever these values exist. One formally may also associate infinite objects, cf. [44]. The numbers obey the quasi shuffle and shuffle algebras of both spaces and a series of additional structural relations. Counting relations of the bases for the different classes of nested sums, iterated integrals and numbers have been derived to a large extent using Witt formulae [137] and their corresponding modifications. The relations, basis representations, argument relations and conversions of the different objects quoted above are implemented in the package HarmonicSums [76.79].

Data analyses are often performed using Mellinspace codes, cf. e.g. [113]. Here the evolution equations can be solved analytically up to a given order in the strong coupling constant. The $N$-space representation for the structure function can be turned into the $x$-space by a single numerical contour integral around the singularities of the problem. This representation requests the sum representations for complex argument $N$. The analytic continuation can be derived applying the shift relations of the sums for $N \rightarrow N+1$ and using their asymptotic representation in analytic form [7, 77, 78, 107]. In the case of the harmonic sums also adaptive numerical representations have been derived [138-140]. The latter may be even obtained in the case of distributions given numerically, cf. e.g. [6].

Data analyses in $x$-space require efficient numerical implementations of the iterated integrals representing the higher order splitting functions and Wilson coefficients. In the case of the harmonic polylogarithms the Bernoulli-improvement [141, 142] allows to map the expressions onto functions like $\log (1 \pm x)$ and their logarithms and polynomials in $x$ within the intervals $[0, \sqrt{2}-1],[\sqrt{2}-1,1]$. The corresponding representations for more involved iterated integrals have still to be worked out. Numerical representations for harmonic polylogarithms were given in [142 145]. Representations for generalized harmonic polylogarithms were derived in [143, 146]. The mathematical structures described in this section could be systematically found calculating the corresponding Feynman integrals in difference fields in $N$-space [65.73], implemented in the package Sigma [63, 64], or using Risch-type algorithms in $x$-space [107, 147]. This also applies for new structures contained in Feynman integrals having not been revealed yet. In this way the growing sets of function spaces describing the Feynman integrals may be uniquely found and explored. Furthermore, for the application of the results in data analyses precise and efficient numerical implementations have to be derived.

\section{Precision Parton Distribution Functions and $\alpha_{s}\left(M_{Z}^{2}\right)$ and $m_{4}^{9}$}

The world data on deep-inelastic scattering and related hard processes at hadron colliders like the Drell-Yan process and jet-production are so precise that NNLO corrections are needed to extract the parton distribution functions, the strong coupling constant $a_{s}\left(\mu^{2}\right)=$ $\alpha_{s}\left(\mu^{2}\right) /(4 \pi)$ and also the charm quark mass $m_{c}$. In the following we give a brief survey on the present status on the determination of these quantities.

The NNLO parton distributions have been determined by five groups (ABM, CT10, JR, MSTW, NNPDF) also resolving the flavor dependence of the sea-quarks.

\footnotetext{
${ }^{9}$ Main results summarized in this section have been worked out by S. Alekhin, J.B., H. Böttcher and S.-O. Moch.
} 


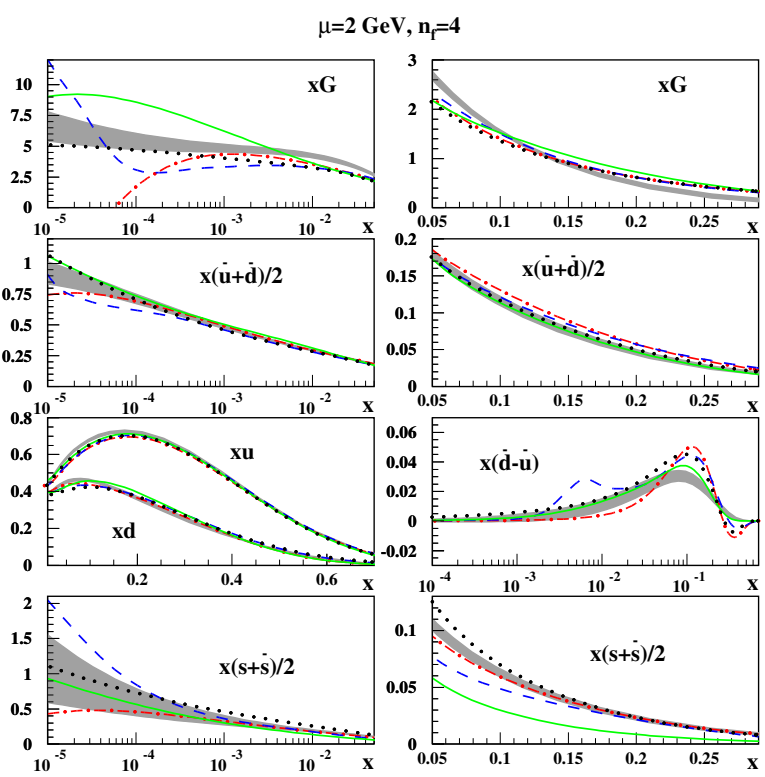

Figure 8: The $1 \sigma$ band for the 4-flavor NNLO ABM12 PDFs [115 at the scale of $\mu=2 \mathrm{GeV}$ versus $x$ obtained in this analysis (shaded area) compared with the ones obtained by other groups (solid lines: JR09 [156], dashed dots: MSTW [148], dashes: NN23 [149], dots: CT10 [150]); from Ref. [115].
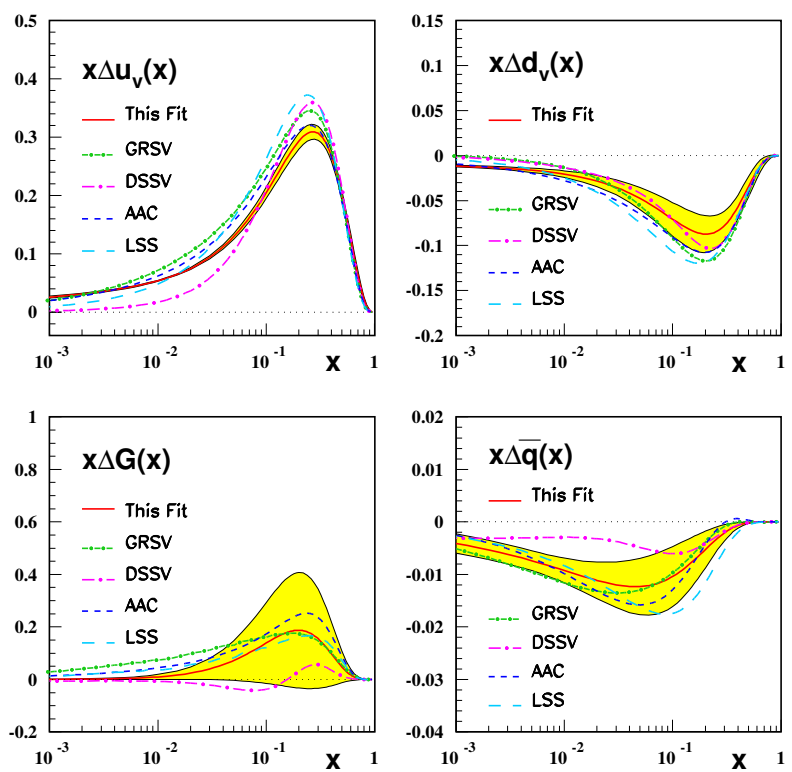

Figure 9: The NLO polarized parton distributions [157] at the input scale $\mu=2 \mathrm{GeV}$ (solid line) compared to results obtained by GRSV (dashed-dotted line) [158], DSSV (long dasheddotted line) [159], AAC (dashed line) [160], and LSS (long dashed line) [161]. The shaded areas represent the fully correlated $1 \sigma$ error bands calculated by Gaussian error propagation; from Ref. [157].

The most recent analyses were given in Refs. [115, 148-
$151{ }^{10}$ A recent comparison has been performed in [115] and the main results are shown in Figure 8 The up- and down quark distributions are well understood. In the case of the gluon distribution there are still significant differences in the small- $x$ region outside the present errors. Here future NNLO analyses of the LHC jet data based on the results of the calculation [154] will lead to a decision. There are still also some significant differences in some analyses for the $\bar{d}-\bar{u}$ distribution, which can be well measured in the Drell-Yan process. The strange quark distribution has the largest errors among the sea quark distributions. Future neutrino and other charged current data will improve it as well as better collider data. For a recent analysis see [155].

In the polarized case so far only NLO analyses have been possible [157-161]. Very recently the NNLO

\begin{tabular}{|c|c|c|}
\hline & $\alpha_{s}\left(M_{Z}^{2}\right)$ & \\
\hline Alekhin [166] & $0.1143 \pm 0.0013$ & \\
\hline BBG [163] & $0.11344_{-0.0021}^{+0.0019}$ & val. analysis, $\mathrm{N}^{2} \mathrm{LO}$ \\
\hline GRS 164 & 0.112 & val. analysis, $\mathrm{N}^{2} \mathrm{LO}$ \\
\hline ABKM [152] & $0.1135 \pm 0.0014$ & HQ: FFNS $N_{F}=3$ \\
\hline JR14 [151] & $0.1136 \pm 0.0004$ & dynamical approach \\
\hline JR14 [151] & $0.1162 \pm 0.0006$ & including NLO-jets \\
\hline MSTW [167] & $0.1171 \pm 0.0014$ & \\
\hline Thorne [168] & 0.1136 & $\mathrm{DIS}+\mathrm{DY}+\mathrm{HT}^{*}$ \\
\hline $\mathrm{ABM}_{1} 1_{J}$ [169] & $\begin{array}{l}0.1134-0.1149 \\
\pm 0.0012\end{array}$ & Tevatron jets NLO \\
\hline ABM12 [115] & $0.1133 \pm 0.0011$ & \\
\hline ABM12 [115] & $0.1132 \pm 0.0011$ & (without jets) \\
\hline CTEQ [150] & $0.1159 \ldots 0.1162$ & \\
\hline CTEQ [150] & 0.1140 & (without jets) \\
\hline NN21 [170] & $0.1174 \pm 0.0006$ & \\
\hline$e^{+} e^{-}$thr. [171] & $0.1131_{-0.0022}^{+0.0028}$ & \\
\hline$e^{+} e^{-}$thr. [172] & $0.1140 \pm 0.0015$ & \\
\hline BBG [163 & $0.1141_{-0.0022}^{+0.0020}$ & val. analysis $\mathrm{N}^{3} \mathrm{LO}$ \\
\hline World Average & $0.1185 \pm 0.0006$ & (2013) [173] \\
\hline
\end{tabular}

Table 1: A survey on the present status on the determination of $\alpha_{s}\left(M_{Z}^{2}\right)$ from the deep-inelastic world data and related data at NNLO and $\mathrm{N}^{3} \mathrm{LO}$.

anomalous dimensions have been calculated [162] and will allow for NNLO analyses in the future. In Figure 9 we compare the present polarized parton distribution functions obtained from the inclusive and semiinclusive world data. While a reasonable agreement is found for the valence quark distributions the present differences are larger in the case of the gluon and sea quark data. In particular the gluon distribution is strongly correlated with the value of the strong coupling constant.

\footnotetext{
${ }^{10}$ For earlier ABM-analyses see Refs. [152, 153].
} 
In this case a NNLO analysis will lead to further improvement. In these analyses proper normalization of the denominator function of the measured asymmetries and the treatment of the higher twist contributions is of importance, since most of the present data stem from the lower $Q^{2}$ domain.

Finally, we discuss the determination of the strong coupling constant $\alpha_{s}\left(M_{Z}^{2}\right)$ from deep inelastic and collider data at NNLO. Valence analyses have been carried out in [163, 164]. Here the gluon uncertainties can be avoided. Sea-quark tail effects were studied in [165], which yield a negligible contribution, however. An early NNLO singlet analysis was carried out in [166].

Again, the higher twist effects have to be dealt with in a careful manner. In the valence analyses one may cut them away for the $\alpha_{s}$ determination and analyze these data separately to determine the higher twist contributions to $F_{2}^{p, d}$, cf. [163, 165, 174]. Today many singlet-analyses deliver comparably low values of $\alpha_{s}$ [115, 150,-152, 166, 168, 169], fully consistent with the results of the non-singlet analyses with values in the range of $0.112-0.114$. Exceptions are the results of MSTW [167] and NNPDF [170]. We would like to note that also the analysis of thrust data in $e^{+} e^{-}$annihilation leads to small values of $\alpha_{s}$ [171, 172] if compared to the present world average. Theoretical uncertainties are due to the heavy flavor treatment of $O(0.0006)[152]$ and higher order effects, comparing the central value in the case of the valence $\mathrm{N}^{3} \mathrm{LO}$ analysis [163] with the value at NNLO of +0.0009 . The reported error of the current world average of $0.0006[173]$ is thus too small. For a survey on other determinations of $\alpha_{s}$ see [175-177]. Low values of $\alpha_{s}$ at NLO have recently been reported from ATLAS and CMS from their jet data [178] with

$$
\begin{aligned}
& \alpha_{s}\left(M_{Z}^{2}\right)=0.111_{-0.0007}^{+0.0017} \\
& \alpha_{s}\left(M_{Z}^{2}\right)=0.1148 \pm 0.0055 .
\end{aligned}
$$

It will be interesting to see the results of the forthcoming NNLO analyses. It is needless to say that both the precise knowledge of $\alpha_{s}\left(M_{Z}\right)$ and the gluon distribution are instrumental for a detailed understanding of the Higgs- and top-quark production cross sections at the LHC [179, 180].

A determination of the charm quark mass from deepinelastic data has been performed in [181], modeling the NNLO effects [111], assigning a corresponding theoretical error. One obtains at NNLO

$m_{c}\left(m_{c}\right)=1.24 \pm 0.03$ (exp) ${ }_{-0.02}^{+0.03}$ (scale) ${ }_{-0.07}^{+0.00} \quad$ (th.) (42)

fully compatible with the result in [182, 183], although with a larger error. The theory error will be further improved after the NNLO heavy flavor corrections of Section 2 are fully available.

\section{Conclusions}

The present world data on deep-inelastic scattering and upcoming collider data from the LHC allow for a very precise determination of the strong coupling constant $\alpha_{s}$ with an accuracy of $1 \%$ accompanied with a precise determination of the parton distribution functions. The corresponding QCD fits require NNLO analyses for which also the heavy flavor corrections have to be known at NNLO. Fortunately, for the structure function $F_{2}\left(x, Q^{2}\right)$ the asymptotic 3-loop corrections can be applied at $Q^{2} \approx 23 \mathrm{GeV}^{2}$ for the charm contributions, which yield the most important part. Recently we made important progress in calculating these corrections, after having completed a series of moments for all quantities in 2009. All logarithmic corrections are known and six out of eight OMEs are completed for general values of $N$ as well as four out of five Wilson coefficients.

On the technical side, the calculation had to be accompanied by various innovative developments in summation and integration theory and new techniques in treating massive 3-loop Feynman diagrams containing local operator insertions. This also has led to the full exploration of new mathematical function spaces. All these techniques are of importance also in other massive calculations, e.g. for processes at the large hadron collider LHC and a forthcoming linear collider like the ILC. The ongoing computations are performed on a number of main frames with now typically $\sim 400$ Gbyte RAM and 20 Tbyte fast discs. With the help of these machines, using parallel processing, we can already reduce all diagrams to master integrals. We still have to complete the calculation of master integrals using the techniques being described in this article for the yet open OMEs and Wilson coefficients. Finally, also the graphs containing two different mass scales have to be calculated to complete all the corrections.

Acknowledgment. For cooperation on the projects reported on we would like to thank J. Ablinger, S. Alekhin, A. Behring, I. Bierenbaum, H. Böttcher, A. Hasselhuhn, S. Klein, A. von Manteuffel, S.O. Moch, C. Raab, M. Round, and F. Wißbrock. We thank K. Chetyrkin and M. Steinhauser for discussions. This work was supported in part by DFG Sonderforschungsbereich Transregio 9, Computergestützte Theoretische Teilchenphysik, for which we would like to thank the Deutsche Forschungsgemeinschaft, the Austrian Science Fund (FWF) grants P20347-N18 and SFB F50 (F5009-N15), and the European Commission through contracts PITN-GA-2010-264564 (LHCPhenoNet) and PITN-GA-2012-316704 (HIGGSTOOLS). 


\section{References}

[1] W. L. van Neerven and E. B. Zijlstra, Phys. Lett. B 272 (1991) 127; Phys. Lett. B 273 (1991) 476; Nucl. Phys. B 383 (1992) 525.

[2] S. Moch, J.A.M. Vermaseren and A. Vogt, Nucl. Phys. B 688 (2004) 101 [hep-ph/0403192].

[3] A. Vogt, S. Moch and J.A.M. Vermaseren, Nucl. Phys. B 691 (2004) 129 [hep-ph/0404111].

[4] J.A.M. Vermaseren, A. Vogt and S. Moch, Nucl. Phys. B 724 (2005) 3 |hep-ph/0504242

[5] E. Laenen, S. Riemersma, J. Smith and W. L. van Neerven, Nucl. Phys. B 392 (1993) 162, 229;

S. Riemersma, J. Smith and W. L. van Neerven, Phys. Lett. B 347 (1995) 143 |hep-ph/9411431|.

[6] S. I. Alekhin and J. Blümlein, Phys. Lett. B 594 (2004) 299 |hep-ph/0404034].

[7] J. Blümlein, Comput. Phys. Commun. 180 (2009) 2218 arXiv:0901.3106 [hep-ph]].

[8] J. Blümlein, S. Klein, C. Schneider and F. Stan, J. Symbolic Comput. 47 (2012) 1267-1289 [arXiv:1011.2656 [cs.SC]].

[9] J. Ablinger and J. Blümlein, arXiv:1304.7071 [math-ph].

[10] J. Ablinger, J. Blümlein and C. Schneider, J. Phys. Conf. Ser. 523 (2014) 012060 [arXiv:1310.5645 [math-ph]].

[11] K. Jansen et al., these Proceedings.

[12] J. Blümlein, Prog. Part. Nucl. Phys. 69 (2013) 28 arXiv:1208.6087 [hep-ph]].

[13] J. Blümlein, V. Ravindran and W. L. van Neerven, Nucl. Phys. B 586 (2000) 349 |hep-ph/0004172].

[14] M. Buza, Y. Matiounine, J. Smith, R. Migneron and W. L. van Neerven, Nucl. Phys. B 472 (1996) 611 |arXiv:hep$\mathrm{ph} / 9601302$.

[15] J. Blümlein, A. De Freitas, W. L. van Neerven and S. Klein, Nucl. Phys. B 755 (2006) 272 [arXiv:hep-ph/0608024].

[16] A. Behring, I. Bierenbaum, J. Blümlein, A. De Freitas, S. Klein and F. Wißbrock, Eur. Phys. J. C 74 (2014) 3033 arXiv:1403.6356 [hep-ph]]

[17] I. Bierenbaum, J. Blümlein and S. Klein, Nucl. Phys. B 820 (2009) 417 [arXiv:0904.3563 [hep-ph]].

[18] E. Witten, Nucl. Phys. B 104 (1976) 445;

J. Babcock, D. W. Sivers and S. Wolfram, Phys. Rev. D 18 (1978) 162;

M. A. Shifman, A. I. Vainshtein and V. I. Zakharov, Nucl. Phys. B 136 (1978) 157 [Yad. Fiz. 27 (1978) 455];

J. P. Leveille and T. J. Weiler, Nucl. Phys. B 147 (1979) 147;

M. Glück, E. Hoffmann and E. Reya, Z. Phys. C 13 (1982) 119

[19] I. Bierenbaum, J. Blümlein and S. Klein, Phys. Lett. B 672 (2009) 401 [arXiv:0901.0669 [hep-ph]].

[20] I. Bierenbaum, J. Blümlein and S. Klein, Phys. Lett. B 648 (2007) 195 |hep-ph/0702265 [HEP-PH]].

[21] I. Bierenbaum, J. Blümlein and S. Klein, Nucl. Phys. B 780 (2007) 40 [hep-ph/0703285].

[22] M. Buza, Y. Matiounine, J. Smith and W. L. van Neerven, Eur. Phys. J. C 1 (1998) 301 [hep-ph/9612398].

[23] M. Buza, Y. Matiounine, J. Smith and W. L. van Neerven, Nucl. Phys. B 485 (1997) 420 |hep-ph/9608342].

[24] I. Bierenbaum, J. Blümlein and S. Klein, arXiv:0706.2738 [hep$\mathrm{ph}$.

[25] M. Buza and W. L. van Neerven, Nucl. Phys. B 500 (1997) 301 [hep-ph/9702242].

[26] J. Blümlein, A. Hasselhuhn and T. Pfoh, Nucl. Phys. B 881 (2014) 1 [arXiv:1401.4352 [hep-ph]].

[27] M. Glück, S. Kretzer and E. Reya, Phys. Lett. B 380 (1996) 171 [Erratum-ibid. B 405 (1997) 391] [hep-ph/9603304].
[28] J. Blümlein, A. Hasselhuhn, P. Kovacikova and S. Moch, Phys. Lett. B 700 (2011) 294 [arXiv:1104.3449 [hep-ph]]

[29] H. D. Politzer, Phys. Rept. 14 (1974) 129; J. Blümlein and N. Kochelev, Phys. Lett. B 381 (1996) 296 |hepph/9603397]; Nucl. Phys. B 498 (1997) 285 [hep-ph/9612318].

[30] I. Bierenbaum, J. Blümlein, S. Klein and C. Schneider, Nucl. Phys. B 803 (2008) 1 |arXiv:0803.0273 [hep-ph]].

[31] J. Ablinger, J. Blümlein, S. Klein, C. Schneider and F. Wißbrock, arXiv:1106.5937 [hep-ph];

J. Ablinger, J. Blümlein, A. Hasselhuhn, S. Klein, C. Schneider and F. Wißbrock, PoS (RADCOR2011) 031 arXiv:1202.2700 [hep-ph]]; J. Blümlein and F. Wißbrock, DESY 14-019.

[32] J. Blümlein and W. L. van Neerven, Phys. Lett. B 450 (1999) 417 [hep-ph/9811351].

[33] K. G. Wilson, Phys. Rev. 179 (1969) 1499; R.A. Brandt and G. Preparata, Fortschr. Phys. 18 (1970) 249; W. Zimmermann, Lect. on Elementary Particle Physics and Quantum Field Theory, Brandeis Summer Inst., Vol. 1, (MIT Press, Cambridge, 1970), p. 395;

Y. Frishman, Annals Phys. 66 (1971) 373.

[34] P. Nogueira, J. Comput. Phys. 105 (1993) 279

[35] M. Tentyukov and J. A. M. Vermaseren, Comput. Phys. Commun. 181 (2010) 1419 [hep-ph/0702279]; J. A. M. Vermaseren, arXiv:math-ph/0010025

[36] T. van Ritbergen, A. N. Schellekens and J. A. M. Vermaseren, Int. J. Mod. Phys. A 14 (1999) 41 [arXiv:hep-ph/9802376].

[37] J. Ablinger, A. Behring, J. Blümlein, A. De Freitas, A. von Manteuffel and C. Schneider, Nucl. Phys. B (2014) in print arXiv:1409.1135 [hep-ph]].

[38] N. Gray, D. J. Broadhurst, W. Grafe and K. Schilcher, Z. Phys. C 48 (1990) 673;

K. G. Chetyrkin and M. Steinhauser, Nucl. Phys. B 573 (2000) 617 |hep-ph/9911434];

K. Melnikov and T. v. Ritbergen, Phys. Lett. B 482 (2000) 99 [hep-ph/9912391]; Nucl. Phys. B 591 (2000) 515 |hep$\mathrm{ph} / 0005131$.

[39] J. Blümlein, A. De Freitas and W. van Neerven, Nucl. Phys. B 855 (2012) 508 [arXiv:1107.4638 [hep-ph]].

[40] L. F. Abbott, Nucl. Phys. B 185 (1981) 189; A. Rebhan, Z. Phys. C 30 (1986) 309;

F. Jegerlehner and O. V. Tarasov, Nucl. Phys. B 549 (1999) 481 arXiv:hep-ph/9809485

[41] M. Steinhauser, Comput. Phys. Commun. 134 (2001) 335 |hepph/0009029.

[42] V. Barone, A. Drago and P. G. Ratcliffe, Phys. Rept. 359 (2002) 1 arXiv:hep-ph/0104283.

[43] J. Blümlein, S. Klein and B. Tödtli, Phys. Rev. D 80 (2009) 094010 [arXiv:0909.1547 [hep-ph]].

[44] J. Blümlein, D.J. Broadhurst and J.A.M. Vermaseren, Comput. Phys. Commun. 181 (2010) 582 |arXiv:0907.2557 [math-ph]].

[45] L. Lewin, Dilogarithms and Associated Functions, (Macdonald, London, 1958)

[46] L. Lewin, Polylogarithms and Associated Functions, (North Holland, New York, 1981).

[47] J. Lagrange, Nouvelles recherches sur la nature et la propagation du son, Miscellanea Taurinensis, t. II, 1760-61; Oeuvres t. I, p. 263;

C.F. Gauss, Theoria attractionis corporum sphaeroidicorum ellipticorum homogeneorum methodo novo tractate, Commentationes societas scientiarum Gottingensis recentiores, Vol III, 1813, Werke Bd. V pp. 5-7;

G. Green, Essay on the Mathematical Theory of Electricity and Magnetism, Nottingham, 1828 [Green Papers, pp. 1-115]; M. Ostrogradski, Mem. Ac. Sci. St. Peters., 6, (1831) 39; 
K. G. Chetyrkin, A. L. Kataev and F. V. Tkachov, Nucl. Phys. B 174 (1980) 345;

F. V. Tkachov, Phys. Lett. B 100 (1981) 65;

K. G. Chetyrkin and F. V. Tkachov, Nucl. Phys. B 192 (1981) 159.

[48] S. Laporta, Int. J. Mod. Phys. A 15 (2000) 5087 hep$\mathrm{ph} / 0102033$.

[49] A. von Manteuffel and C. Studerus, arXiv:1201.4330 [hep-ph]; C. Studerus, Comput. Phys. Commun. 181 (2010) 1293 arXiv:0912.2546 [physics.comp-ph]].

[50] R.H. Lewis, Computer Algebra System Fermat, http://home.bway.net/lewis.

[51] C. W. Bauer, A. Frink and R. Kreckel, Symbolic Computation 33 (2002) 1, cs/0004015 [cs-sc].

[52] C. Anastasiou and A. Lazopoulos, JHEP 0407 (2004) 046 |hepph/0404258];

A. V. Smirnov and V. A. Smirnov, Comput. Phys. Commun. 184 (2013) 2820 [arXiv:1302.5885 [hep-ph]];

A. V. Smirnov, JHEP 0810 (2008) 107 |arXiv:0807.3243 [hep$\mathrm{ph}]]$;

R. N. Lee, arXiv:1212.2685 [hep-ph].

[53] W.N. Bailey, Generalized Hypergeometric Series, (Cambridge University Press, Cambridge, 1935).

[54] L.J. Slater, Generalized Hypergeometric Functions, (Cambridge University Press, Cambridge, 1966).

[55] R. Hamberg, Second Order Gluonic Contributions to Physical Quantities, Ph.D. Thesis, Univ. of Leiden (1991).

[56] P. Appell and J. Kampé de Fériet, Fonctions Hypergéométriques et Hypersphériques, Polynomes D' Hermite, (Gauthier-Villars, Paris, 1926);

P. Appell, Les Fonctions Hypergëométriques de Plusieur Variables, (Gauthier-Villars, Paris, 1925);

J. Kampé de Fériet, La fonction hypergëométrique, (GauthierVillars, Paris, 1937);

H. Exton, Multiple Hypergeometric Functions and Applications, (Ellis Horwood, Chichester, 1976); Handbook of Hypergeometric Integrals, (Ellis Horwood, Chichester, 1978);

H.M. Srivastava and P.W. Karlsson, Multiple Gaussian Hypergeometric Series, (Ellis Horwood, Chicester, 1985).

[57] T. Huber and D. Maitre, Comput. Phys. Commun. 175 (2006) 122 [hep-ph/0507094]; Comput. Phys. Commun. 178 (2008) 755 [arXiv:0708.2443 [hep-ph]].

[58] S. Weinzierl, Comput. Phys. Commun. 145 (2002) 357-370, math-ph/0201011].

[59] S. Weinzierl, J. Math. Phys. 45 (2004) 2656 hep-ph/0402131].

[60] S.-O. Moch and P. Uwer, Comput. Phys. Commun. 174 (2006) 759-770, math-ph/0508008].

[61] M.Y. Kalmykov, B.F.L. Ward and S.A. Yost, JHEP 0710 (2007) 048 [arXiv:0707.3654 [hep-th]].

[62] V. V. Bytev, M. Y. Kalmykov and B. A. Kniehl, Comput. Phys. Commun. 184 (2013) 2332 |arXiv:1105.3565 [math-ph]].

[63] C. Schneider, Sém. Lothar. Combin. 56 (2007) 1, article B56b.

[64] C. Schneider, Computer Algebra in Quantum Field Theory: Integration, Summation and Special Functions Texts and Monographs in Symbolic Computation eds. C. Schneider and J. Blümlein (Springer, Wien, 2013) 325 arXiv:1304.4134 [cs.SC].

[65] M. Karr 1981 J. ACM 28 (1981) 305.

[66] C. Schneider, Symbolic Summation in Difference Fields Ph.D. Thesis RISC, J. Kepler University, Linz technical report 01-17 (2001).

[67] C. Schneider, J. Differ. Equations Appl. 11 (2005) 799.

[68] C. Schneider, J. Algebra Appl. 6 (2007) 415.

[69] C. Schneider, J. Symbolic Comput. 43 (2008) 611. arXiv:0808.2543v1].
[70] C. Schneider, Appl. Algebra Engrg. Comm. Comput. 21 (2010) 1.

[71] C. Schneider, Motives, Quantum Field Theory, and Pseudodifferential Operators (Clay Mathematics Proceedings vol 12) ed Carey A, Ellwood D, Paycha S and Rosenberg S (Amer. Math. Soc) (2010) 285 arXiv:0808.2543

[72] C. Schneider, Ann. Comb. 14 (2010) 533 arXiv:0808.2596); arXiv:1408.2776 [cs.SC]

[73] C. Schneider, in : Lecture Notes in Computer Science (LNCS) eds. J. Guitierrez, J. Schicho, M. Weimann, in press, arXiv:1307.7887 [cs.SC] (2013).

[74] J. Ablinger, J. Blümlein, S. Klein and C. Schneider, Nucl. Phys. Proc. Suppl. 205-206 (2010) 110 |arXiv:1006.4797|[math-ph]]; J. Blümlein, A. Hasselhuhn and C. Schneider, PoS (RADCOR 2011) 032 |arXiv:1202.4303 [math-ph]];

C. Schneider, J. Phys. Conf. Ser. 523 (2014) 012037 arXiv:1310.0160 [cs.SC]].

[75] C. Schneider, Advances in Applied Math. 34(4) (2005) 740; J. Ablinger, J. Blümlein, M. Round and C. Schneider, PoS(LL2012)050, (2012) 14 p. |arXiv:1210.1685 [cs.SC]]; M. Round et al., in preparation.

[76] A Computer Algebra Toolbox for Harmonic Sums Related to Particle Physics, Master's thesis, RISC, J. Kepler University, Linz (2009) arXiv:1011.1176 [math-ph].

[77] J. Ablinger, J. Blümlein and C. Schneider, J. Math. Phys. 52 (2011) 102301 |arXiv:1105.6063 [math-ph]].

[78] J. Ablinger, J. Blümlein and C. Schneider, J. Math. Phys. 54 (2013) 082301 |arXiv:1302.0378 [math-ph]].

[79] J. Ablinger, Computer Algebra Algorithms for Special Functions in Particle Physics, Ph.D. thesis, RISC, J. Kepler University, Linz (2012) arXiv:1305.0687 [math-ph]; PoS LL 2014 (2014) 019 [arXiv:1407.6180 [cs.SC]].

[80] E.W. Barnes, Proc. Lond. Math. Soc. (2) 6 (1908) 141; Quart. Journ. Math. 41 (1910) 136; H. Mellin, Math. Ann. 68 (1910) 305.

[81] M. Czakon, Comput. Phys. Commun. 175 (2006) 559 |hep$\mathrm{ph} / 0511200$.

[82] A. V. Smirnov and V. A. Smirnov, Eur. Phys. J. C 62 (2009) 445 arXiv:0901.0386 [hep-ph]].

[83] K. Wegschaider, Computer generated proofs of binomial multisum identities, Master's thesis, RISC, J. Kepler University (1997).

[84] M. Apagodu and D. Zeilberger, Adv. Appl. Math. (Special Regev issue), 37 (2006) 139.

[85] A. V. Kotikov, Phys. Lett. B 254 (1991) 158;

M. Caffo, H. Czyz, S. Laporta and E. Remiddi, Acta Phys. Polon. B 29 (1998) 2627 |hep-th/9807119|;

Nuovo Cim. A 111 (1998) 365 [hep-th/9805118];

T. Gehrmann and E. Remiddi, Nucl. Phys. B 580 (2000) 485 hep-ph/9912329];

M. Caffo, H. Czyz and E. Remiddi, Nucl. Phys. B 634 (2002) 309 [hep-ph/0203256].

[86] S. Gerhold, Uncoupling systems of linear Ore operator equations, Master's thesis, RISC, J. Kepler University, Linz, 2002.

[87] F. Brown, Commun. Math. Phys. 287 (2009) 925 arXiv:0804.1660 [math.AG]].

[88] J. Ablinger, J. Blümlein, C. Raab, C. Schneider and F. Wißbrock, Nucl. Phys. B 885 (2014) 409 arXiv:1403.1137 [hep-ph]].

[89] J. Ablinger, J. Blümlein, A. Hasselhuhn, S. Klein, C. Schneider and F. Wissbrock, Nucl. Phys. B 864 (2012) 52 arXiv:1206.2252 [hep-ph]].

[90] M. Kauers, Guessing Handbook, Technical Report RISC 09-07 (2009) JKU Linz.

[91] J. Blümlein, M. Kauers, S. Klein and C. Schneider, Comput. 
Phys. Commun. 180 (2009) 2143 [arXiv:0902.4091 [hep-ph]].

[92] S. A. Larin, T. van Ritbergen and J. A. M. Vermaseren, Nucl. Phys. B 427 (1994) 41;

S. A. Larin, P. Nogueira, T. van Ritbergen and J. A. M. Vermaseren, Nucl. Phys. B 492 (1997) 338 |arXiv:hep$\mathrm{ph} / 9605317$;

A. Retey and J. A. M. Vermaseren, Nucl. Phys. B 604 (2001) 281 [arXiv:hep-ph/0007294].

[93] J. Ablinger, J. Blümlein, A. De Freitas, A. Hasselhuhn, A. von Manteuffel, M. Round, C. Schneider and F. Wißbrock, Nucl. Phys. B 882 (2014) 263 [arXiv:1402.0359 [hep-ph]].

[94] J. Ablinger, A. Behring, J. Blümlein, A. De Freitas, A. Hasselhuhn, A. von Manteuffel, M. Round, C. Schneider, and F. Wißbrock, Nucl. Phys. B 886 (2014) 733 |arXiv:1406.4654 [hep-ph]].

[95] V. N. Velizhanin, Nucl. Phys. B 864 (2012) 113 [arXiv:1203.1022 [hep-ph]].

[96] E. G. Floratos, D. A. Ross and C. T. Sachrajda, Nucl. Phys. B 129 (1977) 66 [Erratum-ibid. B 139 (1978) 545];

A. Gonzalez-Arroyo, C. Lopez and F. J. Yndurain, Nucl. Phys. B 153 (1979) 161;

G. Curci, W. Furmanski and R. Petronzio, Nucl. Phys. B 175 (1980) 27;

E. G. Floratos, C. Kounnas and R. Lacaze, Nucl. Phys. B 192 (1981) 417;

S. Moch and J. A. M. Vermaseren, Nucl. Phys. B 573 (2000) 853 [hep-ph/9912355].

[97] S. Kumano and M. Miyama, Phys. Rev. D 56 (1997) 2504 [arXiv:hep-ph/9706420];

W. Vogelsang, Phys. Rev. D 57 (1998) 1886 |arXiv:hep$\mathrm{ph} / 9706511]$ and references therein;

A. Hayashigaki, Y. Kanazawa and Y. Koike, Phys. Rev. D 56 (1997) 7350 [hep-ph/9707208].

[98] J. Blümlein and J. A. M. Vermaseren, Phys. Lett. B 606 (2005) 130 [hep-ph/0411111].

[99] J. A. Gracey, Nucl. Phys. B 662 (2003) 247 arXiv:hepph/0304113]; Nucl. Phys. B 667 (2003) 242 |arXiv:hepph/0306163]; JHEP 0610 (2006) 040 [arXiv:hep-ph/0609231]; Phys. Lett. B 643 (2006) 374 arXiv:hep-ph/0611071|.

[100] A. A. Bagaev, A. V. Bednyakov, A. F. Pikelner and V. N. Velizhanin, Phys. Lett. B 714 (2012) 76 arXiv:1206.2890 [hep-ph]].

[101] J. Ablinger, J. Blümlein, S. Klein, C. Schneider and F. Wißbrock, Nucl. Phys. B 844 (2011) 26 arXiv:1008.3347 [hep-ph]].

[102] J. Blümlein, A. Hasselhuhn, S. Klein and C. Schneider, Nucl. Phys. B 866 (2013) 196 [arXiv:1205.4184 [hep-ph]].

[103] A. Behring, J. Blümlein, A. De Freitas, T. Pfoh, C. Raab, M. Round, J. Ablinger and A. Hasselhuhn et al., PoS RADCOR 2013 (2013) 058 [arXiv:1312.0124 [hep-ph]].

[104] J. Ablinger, J. Blümlein, A. De Freitas, A. Hasselhuhn, A. von Manteuffel, M. Round and C. Schneider, Nucl. Phys. B 885 (2014) 280 [arXiv:1405.4259 [hep-ph]].

[105] J. A. M. Vermaseren, Int. J. Mod. Phys. A 14 (1999) 2037/hep$\mathrm{ph} / 9806280$.

[106] J. Blümlein and S. Kurth, Phys. Rev. D 60 (1999) 014018 [hep$\mathrm{ph} / 9810241]$.

[107] J. Ablinger, J. Blümlein, C. G. Raab and C. Schneider, J. Math. Phys. (2014) in print arXiv:1407.1822 [hep-th]].

[108] S. Moch, P. Uwer and S. Weinzierl, J. Math. Phys. 43 (2002) 3363 [hep-ph/0110083].

[109] E. Remiddi and J. A. M. Vermaseren, Int. J. Mod. Phys. A 15 (2000) 725 [hep-ph/9905237].

[110] J. C. Ward, Phys. Rev. 78 (1950) 182; Y. Takahashi, Nuovo Cim. 6 (1957) 371.
[111] H. Kawamura, N. A. Lo Presti, S. Moch and A. Vogt, Nucl. Phys. B 864 (2012) 399 [arXiv:1205.5727 [hep-ph]].

[112] S. Catani, M. Ciafaloni and F. Hautmann, Nucl. Phys. B 366 (1991) 135.

[113] J. Blümlein and A. Vogt, Phys. Rev. D 58 (1998) 014020 [hep$\mathrm{ph} / 9712546$.

[114] J. Blümlein and A. Vogt, Phys. Lett. B 370 (1996) 149 |hepph/9510410]; Phys. Lett. B 386 (1996) 350 [hep-ph/9606254]; J. Blümlein, In : New trends in HERA physics (1999) 42 |hep$\mathrm{ph} / 9909449$.

[115] S. Alekhin, J. Blümlein and S. Moch, Phys. Rev. D 89 (2014) 054028 arXiv:1310.3059 [hep-ph]].

[116] S. Alekhin, G. Altarelli, N. Amapane, J. Andersen, V. Andreev, M. Arneodo, V. Avati and J. Baines et al., hep-ph/0601012 hep$\mathrm{ph} / 0601013$

Z. J. Ajaltouni, S. Albino, G. Altarelli, F. Ambroglini, J. Anderson, G. Antchev, M. Arneodo and P. Aspell et al., arXiv:0903.3861 [hep-ph].

[117] D. Boer, M. Diehl, R. Milner, R. Venugopalan, W. Vogelsang, D. Kaplan, H. Montgomery and S. Vigdor et al., arXiv:1108.1713 [nucl-th].

[118] A. Accardi et al. arXiv:1212.1701 [nucl-ex].

[119] J. L. Abelleira Fernandez et al. [LHeC Study Group Collaboration], J. Phys. G 39 (2012) 075001 arXiv:1206.2913 [physics.acc-ph]].

[120] R. Harlander, T. Seidensticker, M. Steinhauser, Phys. Lett. B426 (1998) 125, |hep-ph/9712228|; T. Seidensticker, [hep-ph/9905298].

[121] S. Laporta and E. Remiddi, Phys. Lett. B 379 (1996) 283 hep$\mathrm{ph} / 9602417]$.

[122] R. Hamberg, W. L. van Neerven and T. Matsuura, Nucl. Phys. B 359 (1991) 343 [Erratum-ibid. B 644 (2002) 403].

[123] J. Blümlein and V. Ravindran, Nucl. Phys. B 716 (2005) 128 hep-ph/0501178].

[124] J. Blümlein and V. Ravindran, Nucl. Phys. B 749 (2006) 1 [hep-ph/0604019].

[125] D. J. Broadhurst and D. Kreimer, Phys. Lett. B 393 (1997) 403 |hep-th/9609128].

[126] J. M. Borwein, D. M. Bradley, D. J. Broadhurst and P. Lisonek, Trans. Am. Math. Soc. 353 (2001) 907 [math/9910045] [mathcal].

[127] N. Nielsen, Nova Acta Leopoldoldina, Halle, XC (1909) 121.

[128] K. S. Kölbig, J. A. Mignoco and E. Remiddi, BIT Numerical Mathematics 10 (1970) 38

[129] K. S. Kölbig, SIAM J. Math. Anal. 17 (1986) 1232.

[130] A. Devoto and D. W. Duke, Riv. Nuovo Cim. 7N6 (1984) 1

[131] D. J. Broadhurst, Eur. Phys. J. C 8 (1999) 311 |hepth/9803091.

[132] J. Fleischer, A.V. Kotikov and O.L. Veretin, Nucl. Phys. B 547 (1999) 343 |hep-ph/9808242|;

M.Y. Kalmykov and O. Veretin, Phys. Lett. B 483 (2000) 315 hep-th/0004010];

A.I. Davydychev and M.Y. Kalmykov, Nucl. Phys. B 699 (2004) 3 [hep-th/0303162].

[133] A. B. Goncharov. Math. Res. Lett., 5 (1998) 497 arXiv:1105.2076 [math.AG]]

[134] C. Reutenauer, Free Lie algebras. (Oxford, University Press, 1993).

[135] J. Blümlein, Comput. Phys. Commun. 159 (2004) 19 |hep$\mathrm{ph} / 0311046$

[136] J. Blümlein, Proceedings of the Workshop "Motives, Quantum Field Theory, and Pseudodifferential Operators", held at the Clay Mathematics Institute, Boston University, June 2-13, 2008, Clay Mathematics Proceedings 12 (2010) 167, Eds. A. Carey, D. Ellwood, S. Paycha, S. Rosenberg, 
arXiv:0901.0837 [math-ph].

[137] E. Witt, Journ. Reine und Angew. Mathematik, 177 (1937) 152; Journ. Reine und Angew. Mathematik, 177 (1937) 152.

[138] J. Blümlein, Comput. Phys. Commun. 133 (2000) 76 |hep$\mathrm{ph} / 0003100$.

[139] J. Blümlein and S. O. Moch, Phys. Lett. B 614 (2005) 53 [hep$\mathrm{ph} / 0503188$.

[140] A. V. Kotikov and V. N. Velizhanin, hep-ph/0501274

[141] L. Euler, Commentarii academiae scientiarum Petropolitane 6 (1738) 68;

C. MacLaurin, Treatise of Fluxions, (T.W. and T. Ruddimans, Edinburgh, 1742);

G. 't Hooft and M. J. G. Veltman, Nucl. Phys. B 153 (1979) 365; P. Debye, Ann. Phys. (Leipzig) 344 (1912) 789.

[142] T. Gehrmann and E. Remiddi, Comput. Phys. Commun. 141 (2001) 296 [hep-ph/0107173].

[143] J. Vollinga and S. Weinzierl, Comput. Phys. Commun. 167 (2005) 177 |hep-ph/0410259|

[144] D. Maitre, Comput. Phys. Commun. 183 (2012) 846 [hepph/0703052 [hep-ph]]; Comput. Phys. Commun. 174 (2006) 222 [hep-ph/0507152].

[145] S. Buehler and C. Duhr, Comp. Phys. Commun. 185 (2014) 2703 [arXiv: 1106.5739 [hep-ph]].

[146] T. Gehrmann and E. Remiddi, Comput. Phys. Commun. 144 (2002) 200 |hep-ph/0111255|.

[147] R.H. Risch, Trans. Amer. Math. Soc. 139 (1969) 167; M. Bronstein, Symbolic Integration I - Transcendental Functions, $2^{\text {nd }}$ ed. (Springer, Berlin, 2005);

C.G. Raab, Definite Integration in Differential Fields, $\mathrm{PhD}$ Thesis, Johannes Kepler Universität Linz, Austria, 2012.

[148] A. D. Martin, W. J. Stirling, R. S. Thorne and G. Watt, Eur. Phys. J. C 63 (2009) 189 [arXiv:0901.0002 [hep-ph]].

[149] R. D. Ball et al., Nucl. Phys. B 867 (2013) 244 arXiv:1207.1303 [hep-ph]

[150] J. Gao et al., Phys. Rev. D 89 (2014) 033009 arXiv:1302.6246 [hep-ph]].

[151] P. Jimenez-Delgado and E. Reya, Phys. Rev. D 89 (2014) 074049 [arXiv: 1403.1852 [hep-ph]].

[152] S. Alekhin, J. Blümlein, S. Klein and S. Moch, Phys. Rev. D 81 (2010) 014032 [arXiv:0908.2766 [hep-ph]].

[153] S. Alekhin, J. Blümlein and S. Moch, Phys. Rev. D 86 (2012) 054009 [arXiv: 1202.2281 [hep-ph]].

[154] J. Currie, A. Gehrmann-De Ridder, E. W. N. Glover and J. Pires, JHEP 1401 (2014) 110 [arXiv:1310.3993 [hep-ph]]; A. Gehrmann-De Ridder, T. Gehrmann, E. W. N. Glover and J. Pires, Phys. Rev. Lett. 110 (2013) 16, 162003 arXiv:1301.7310 [hep-ph]].

[155] S. Alekhin, J. Blümlein, L. Caminadac, K. Lipka, K. Lohwasser, S. Moch, R. Petti and R. Placakyte, arXiv:1404.6469 [hep-ph].

[156] P. Jimenez-Delgado and E. Reya, Phys. Rev. D 79 (2009) 074023 arXiv:0810.4274 [hep-ph]]

[157] J. Blümlein and H. Böttcher, Nucl. Phys. B 841 (2010) 205 arXiv:1005.3113 [hep-ph]].

[158] M. Glück, E. Reya, M. Stratmann and W. Vogelsang, Phys. Rev. D 63 (2001) 094005 [arXiv:hep-ph/0011215].

[159] D. de Florian, R. Sassot, M. Stratmann and W. Vogelsang, Phys. Rev. Lett. 101 (2008) 072001 arXiv:0804.0422 [hepph]]; Phys. Rev. D 80 (2009) 034030 [arXiv:0904.3821 [hep$\mathrm{ph}]]$.

[160] Y. Goto et al. [Asymmetry Analysis collaboration], Phys. Rev. D 62 (2000) 034017 [arXiv:hep-ph/0001046];

M. Hirai, S. Kumano and N. Saito [Asymmetry Analysis Collaboration], Phys. Rev. D 69 (2004) 054021 |arXiv:hepph/0312112]; 74 (2006) 014015 arXiv:hep-ph/0603213];
M. Hirai and S. Kumano [Asymmetry Analysis Collaboration], Nucl. Phys. B 813 (2009) 106 arXiv:0808.0413 [hep-ph]l.

[161] E. Leader, A. V. Sidorov and D. B. Stamenov, Eur. Phys. J. C 23 (2002) 479 |arXiv:hep-ph/0111267]; Phys. Rev. D 73 (2006) 034023 [hep-ph/0512114].

[162] S. Moch, J. A. M. Vermaseren and A. Vogt, arXiv:1409.5131 [hep-ph].

[163] J. Blümlein, H. Böttcher and A. Guffanti, Nucl. Phys. B 774 (2007) 182 |hep-ph/0607200|; Nucl. Phys. Proc. Suppl. 135 (2004) 152 [hep-ph/0407089].

[164] M. Glück, E. Reya and C. Schuck, Nucl. Phys. B 754 (2006) 178 [hep-ph/0604116]

[165] J. Blümlein and H. Böttcher, arXiv:1207.3170 [hep-ph].

[166] S. I. Alekhin, Phys. Lett. B 519 (2001) 57 [hep-ph/0107197].

[167] A. D. Martin, W. J. Stirling, R. S. Thorne, G. Watt, Eur. Phys. J. C64 (2009) 653. arXiv:0905.3531 [hep-ph]].

[168] R.S. Thorne, Eur. Phys. J. C 74 (2014) 2958 arXiv:1402.3536 [hep-ph]].

[169] S. Alekhin, J. Blümlein and S. O. Moch, PoS DIS 2010 (2010) 021 [arXiv:1007.3657[hep-ph]].

[170] R. D. Ball et al. Phys. Lett. B 707 (2012) 66 arXiv:1110.2483 [hep-ph]].

[171] T. Gehrmann, G. Luisoni and P. F. Monni, Eur. Phys. J. C 73 (2013) 2265 [arXiv:1210.6945 [hep-ph]].

[172] R. Abbate, M. Fickinger, A. H. Hoang, V. Mateu and I. W. Stewart, Phys. Rev. D 86 (2012) 094002 arXiv:1204.5746 [hep-ph]].

[173] K.A. Olive et al. (Particle Data Group), Chin. Phys. C, 38, 090001 (2014).

[174] J. Blümlein and H. Böttcher, Phys. Lett. B 662 (2008) 336 arXiv:0802.0408 [hep-ph]].

[175] S. Bethke Workshop on Precision Measurements of $\alpha_{s}$, arXiv:1110.0016 [hep-ph].

[176] S. Moch, S. Weinzierl, S. Alekhin, J. Blümlein, L. de la Cruz, S. Dittmaier, M. Dowling and J. Erler et al., arXiv:1405.4781 [hep-ph].

[177] U. Wolf, these Proceeedings

[178] K. Rabbertz [ATLAS and CMS Collaborations], arXiv: 1312.5694 [hep-ex].

[179] S. Alekhin, J. Blümlein, P. Jimenez-Delgado, S. Moch and E. Reya, Phys. Lett. B 697 (2011) 127 arXiv:1011.6259 [hep$\mathrm{ph}]]$.

[180] S. Alekhin, J. Blümlein and S. Moch, Eur. Phys. J. C 71 (2011) 1723 [arXiv:1101.5261 [hep-ph]].

[181] S. Alekhin, J. Blümlein, K. Daum, K. Lipka and S. Moch, Phys. Lett. B 720 (2013) 172 [arXiv:1212.2355 [hep-ph]].

[182] K.G. Chetyrkin, J.H. Kühn, A. Maier, P. Maierhofer, P. Marquard, M. Steinhauser and C. Sturm, Phys. Rev. D 80 (2009) 074010 [arXiv:0907.2110 [hep-ph]].

[183] J.H. Kühn et al., these Proceeedings. 